\title{
Droplet impingement and wetting behavior on a chemically heterogeneous surface in the Beyond-Cassie-Baxter regime
}

\author{
Guina $\mathrm{Yi}^{\mathrm{a}, \mathrm{b}}$, Ziqi Cai ${ }^{\mathrm{a}, \mathrm{b}, \mathrm{e}, *}$, Zhengming Gao ${ }^{\mathrm{a}, \mathrm{b},{ }^{*}}$, Zhichao Jiang $^{\mathrm{d}}$, Xiongbin Huang ${ }^{\mathrm{a}, \mathrm{b}}$, J.J. Derksen ${ }^{\mathrm{c}}$ \\ ${ }^{a}$ Beijing Advanced Innovation Center for Soft Matter Science and Engineering, Beijing University of Chemical Technology, Beijing 100029, \\ China
}

${ }^{\mathrm{b}}$ State Key Laboratory of Chemical Resource Engineering, School of Chemical Engineering, Beijing University of Chemical Technology, Beijing 100029, China

${ }^{\mathrm{c}}$ School of Engineering, University of Aberdeen, Aberdeen AB24 3UE, UK

${ }^{\mathrm{d}}$ Research Institute of Petroleum Processing, SINOPEC, Beijing 100083, China

${ }^{\mathrm{e}}$ Department of Chemical Science and Engineering, Kobe University, 1-1 Rokkodai, Nada, Hyogo, Kobe 657-8501, Japan

*Corresponding authors. Tel: +86-10-6441-9171; Fax: +86-10-6444-9862. E-mail address: caiziqi@ mail.buct.edu.cn (Ziqi Cai),

gaozm@mail.buct.edu.cn (Zhengming Gao).

\section{AIChE Journal - accepted May 2020}

\begin{abstract}
Droplet impingement and anisotropic wetting on chemically heterogeneous stripe-patterned surfaces is simulated by means of many-body dissipative particle dynamics (MDPD). The ratio of the stripe width and initial droplet diameter, defined as $\beta$, ranges from 0.5 to 1.0 so that the wetting process is in the Beyond-Cassie-Baxter regime and is highly anisotropic. At zero Weber number (that is, without considering droplet inertia) and with superhydrophobic stripes, $\beta$ is the only factor affecting the droplet perpendicular contact angle and aspect ratio. For inertial droplets, $\beta$ and the Weber number are found to have an effect on the eventual droplet morphology on multi-striped surfaces. These morphologies include elongated shape, split-off, and "butterfly" shape. A correlation for critical split-off conditions has been determined. An energy analysis of droplet impingement shows that the normalized surface energy of the droplet is independent of the Weber number if the droplet is elongated or butterfly-shaped.
\end{abstract}

Keywords: Anisotropic wetting, droplet impingement, many-body dissipative particle dynamics, droplet evolution, surface energy 


\section{Introduction}

Anisotropic wetting of a droplet on grooved surfaces ${ }^{1,2}$ and chemically heterogeneous surfaces $^{3-7}$ is commonly observed in nature ${ }^{3}$ and industrial applications, such as ink jet printing ${ }^{4}$, microfluidics systems ${ }^{5}$, droplet evaporation ${ }^{6}$ and cell culture ${ }^{7}$. Different from wetting on homogeneous surfaces, the droplet morphology will adapt to the alternations of wettability on the chemically heterogeneous surface, and the droplet's final shape strongly depends on the topology of wettability of the surface. Various chemically heterogeneous surfaces such as stripes $^{8-10}$, square ${ }^{11,12}$, and triangular patterns ${ }^{13}$ have been investigated. Striped surfaces get a lot of attention due to their relatively easy fabrication. When a droplet deposits on a striped surface slowly, the contact angles of the droplet in the directions perpendicular $\left(\theta_{\perp}\right)$ and parallel $\left(\theta_{\|}\right)$to the stripes can be very different, as shown in Figure 1.

The aspect ratio, W/L as shown in Figure 1, is used to describe the droplet deformation, where $W$ is the width of the droplet perpendicular to the stripes and $L$ is the length of the droplet parallel to the stripes. Relative hydrophobicity of the stripes is described as $\alpha=\frac{W_{b i c}}{W_{\text {lic }}}$, where $W_{\text {bic }}$ and $W_{\text {lic }}$ are the width of hydrophobic and hydrophilic stripe, respectively ${ }^{14}$. Much research has been done for droplet sizes much larger than the stripe width so that the droplet covers multiple stripes simultaneously, typically more than ten ${ }^{15}$. Bliznyuk et al. ${ }^{14,16}$ deposited glycerol droplets of millimeter size on alternating hydrophilic and hydrophobic stripes to investigate the final shape of the droplet. Two spreading regimes ${ }^{16}$ were observed during the droplet deposition. At the beginning of the deposition, droplet spreading is in the inertial regime, where the spreading rate of the droplet in both perpendicular and parallel direction are the same. Subsequently, the droplet is elongated in the viscous regime, where the droplet spreads more along the stripe because of the energy barrier in the perpendicular direction. In addition, they noticed that the contact angles and aspect ratio of the equilibrium droplet only depend on $\alpha$ if the size of the droplet is one to two orders of magnitude larger than the width of the stripes ${ }^{14}$. The experiment ${ }^{14}$ 
was later compared with the simulations conducted by Jansen et al. ${ }^{17}$ based on a finite element method. Both the experiments and simulations indicated a more elongated droplet on the surface with low $\alpha$. Additionally, $\theta_{\perp}$ is close to the intrinsic contact angle of the hydrophobic stripes $\left(\theta_{\mathrm{bic}}\right)$ for a large $\alpha$ range, and $\theta_{\|}$can be modeled by the Cassie-Baxter equation: ${ }^{14}$ $\theta_{\|}=\arccos \left[\frac{\alpha \cos \left(\theta_{\text {bic }}\right)+\cos \left(\theta_{\text {lic }}\right)}{1+\alpha}\right]$, where $\theta_{\text {lic }}$ is the intrinsic contact angle of the hydrophilic stripes. The equation indicates that the morphology of a droplet in the Cassie-Baxter regime can be adjusted by modifying $\theta_{\text {bic }}, \theta_{\text {lic }}$, and $\alpha$.

Different from a droplet spanning multiple stripes, when the stripe width is comparable with the initial droplet radius, the contact angles of the droplet cannot be predicted by the Cassie-Baxter equation anymore ${ }^{18}$. Wang et al. ${ }^{18}$ suggested 0.1 as the critical value for the ratio of stripe width to droplet radius that separates the Cassie-Baxter regime and the Beyond-Cassie-Baxter regime. In the Beyond-Cassie-Baxter regime, the equilibrium shape of the droplet is related to the number of wetted stripes and the initial deposition position. Jansen et al. ${ }^{19}$ defined a scaled radius to describe the relative size of the droplet and the stripes. They performed lattice-Boltzmann simulations to model droplet wetting on striped surfaces with the scaled radius ranging from 0.5 to 1.25 . Interestingly, $\theta_{\perp}$ and the aspect ratio of the droplet do not change monotonically as the scaled radius increases. Instead, they firstly increase until they undergo a sudden transition where a minimum occurs and then they start a new stage of increase. If the number of wetted stripes reduces to three, "lozenge" and "butterfly" shapes are identified when a droplet is deposited on the middle of a hydrophobic and hydrophilic stripe, respectively ${ }^{18}$ ${ }^{20}$. Lipowsky et al. ${ }^{21,22}$ observed four morphologies on a single hydrophilic stripe, where a long liquid channel could be obtained by adding more and more liquid onto a stripe with wettability if $\theta_{\text {lic }}<38^{\circ}$

When the initial velocity of the droplet is significant, the effects of its inertia on the impinging and wetting process cannot be ignored. The impinging dynamics is characterized by the droplet 
Weber number $\left(W e=\frac{\rho U^{2} D_{0}}{\sigma}\right)$, which is the ratio of inertial force to capillary force. Here, $U$ is the impinging velocity; $\rho, \sigma$ and $D_{0}$ are the density, surface tension and initial diameter of the spherical droplet, respectively. Song et al. $^{23}$ found that the droplet could be split off by impinging it on a slim superhydrophobic stripe coating placed on a hydrophilic surface if $W e>20$. In the case of multiple stripes, Zhao et al. ${ }^{24}$ conducted a 2D simulation based on MDPD to study the droplet impingement on a multi-striped surface, and they observed "stick-slip" motion of the droplet during the recoiling stage, which was strongly affected by $W e$ and $\alpha$. Jansen et al. ${ }^{8,25}$ pointed out that more spherical droplets could be obtained in higher We cases. With increasing We, there may be some residues or satellite droplet on hydrophilic stripes. Wang et al. ${ }^{26}$ found that these residues were distributed in circular regions. Zou et al. ${ }^{27}$ indicated that the number of satellite droplets increases with increasing droplet impinging velocity, i.e. with increasing $W e$.

Despite that much work has been performed on the droplet impingement on stripe pattern surfaces, there is no clear understanding of the droplet spreading and impingement behavior in the Beyond-Cassie-Baxter regime. In this regime, the droplet can be deformed, migrate and even be split into two droplets, dependent on stripes width and wettability. The aim of this work is to investigate the droplet behavior in the Beyond-Cassie-Baxter regime characterized by droplet spreading diameters, contact angles, aspect ratios and droplet energy budgets. In this work, the relative stripe width, $\beta$, defined by the ratio between the stripe width $W_{\mathrm{s}}$ and initial droplet diameter $D_{0}$, ranges from 0.5 to 1.0 . The hydrophilic contact angle has been fixed at $45^{\circ}$ and hydrophobic contact angle is variable.

Here we use many-body dissipative particle dynamics (MDPD) to investigate single droplet impingement and wetting behavior. MDPD is a mesoscopic numerical method that has been widely employed in multi-fluid systems with free liquid/vapor or liquid/liquid interfaces, such as capillary flow $^{28}$, liquid droplets on surfaces ${ }^{29-31}$, and bubble formation ${ }^{32}$. All the simulations in 
this work are implemented by a modified MDPD code in the open source molecular dynamics code LAMMPS (Large-scale Atomic/Molecular Massively Parallel Simulator) ${ }^{33}$.

The remainder of the paper is organized along the following lines: a brief introduction about the MDPD method and validation tests are presented in Section 2, and then the results are shown in Section 3. Firstly, we discuss wetting without taking into account inertial effect. We analyze the effects of deposition position and the hydrophobic stripes wettability on the droplet shape. Next, the droplet impingement behavior including inertial effects is discussed. The effects of the stripe arrangement, $\beta$ and $W e$ on droplet morphology are investigated. In addition, the time evolution of droplet surface energy and kinetic energy are introduced to describe the droplet impingement behavior. A final section summarizes the main conclusions and makes suggestions for future work.

\section{Numerical method}

\subsection{MDPD method}

The MDPD method, a mesoscopic numerical method, is derived from dissipative particle dynamics (DPD) $)^{34-36}$. As is the case for the standard DPD method, each particle in MDPD represents a cluster of atoms or molecules and the motion of each particle obeys Newton's second law, where the total force acting on each particle includes conservative force $\mathbf{F}_{\mathrm{ij}}^{\mathbf{C}}$, dissipative force $\mathbf{F}_{\mathbf{i j}}^{\mathbf{D}}$ and random force $\mathbf{F}_{\mathbf{i j}}^{\mathbf{R}}$. Different from standard DPD, an attractive term is introduced in MDPD to simulate vapor-liquid coexistence. For this reason, the conservative force $\left(\mathbf{F}_{\mathbf{i j}}^{\mathbf{C}}\right)$ in MDPD is defined as:

$$
\mathbf{F}_{\mathbf{i j}}^{\mathbf{C}}=\left[A_{i j} w_{C}\left(r_{i j}\right)+B_{i j}\left(\bar{\rho}_{i}+\bar{\rho}_{j}\right) w_{d}\left(r_{i j}\right)\right] \mathbf{e}_{\mathrm{ij}}
$$

Here, $A_{i j}$, usually negative, is the coefficient of the attractive force between particle $i$ and particle $j$ within a cutoff range $r_{C} ; B_{i j}$, usually greater than zero, is the density-dependent repulsive force coefficient within a cutoff range $r_{d}$. The weight functions are $w_{C}\left(r_{i j}\right)=1-r_{i j} / r_{C}$ 
and $w_{d}\left(r_{i j}\right)=1-r_{i j} / r_{d}$ with $r_{i j}$ the distance between particle $i$ and particle $j$, and $\mathbf{e}_{\mathbf{i j}}$ is the unit vector from particle $j$ to $i$. The local density $\rho$ at the location of particle $i$ can be obtained by $\bar{\rho}_{i}=\sum_{j \neq i} w_{\rho}\left(r_{i j}\right)$ where $w_{\rho}\left(r_{i j}\right)=\frac{15}{2 \pi r_{d}^{3}}\left(1-\frac{r_{i j}}{r_{d}}\right)^{2}$, according to Ref. 34 and Ref. 37 .

Apart from the conservative force, the other components in the total force are the same as those in standard DPD. They can be written as:

$$
\begin{gathered}
\mathbf{F}_{\mathbf{i j}}^{\mathbf{D}}=-\gamma w_{D}\left(r_{i j}\right)\left(\mathbf{e}_{\mathbf{i j}} \cdot \mathbf{v}_{\mathbf{i j}}\right) \mathbf{e}_{\mathbf{i j}} \\
\mathbf{F}_{\mathbf{i j}}^{\mathbf{R}}=\delta w_{R}\left(r_{i j}\right) \xi_{i j} \Delta t^{-1 / 2} \mathbf{e}_{\mathbf{i j}}
\end{gathered}
$$

where $\gamma$ and $\delta$ are the friction coefficient and the noise amplitude, respectively; $w_{D}$ and $w_{R}$ are weight functions; $\mathbf{v}_{\mathbf{i j}}=\mathbf{v}_{\mathbf{i}}-\mathbf{v}_{\mathbf{j}}$ is the relative velocity between particle $i$ and particle $j$; $\xi_{i j}$ is a random Gaussian number with zero mean and unit variance. To satisfy the fluctuation-dissipation theorem requires that $\delta^{2}=2 \gamma k_{B} T$ and $w_{D}\left(r_{i j}\right)=\left[w_{R}\left(r_{i j}\right)\right]^{2}$, where $k_{B}$ is the Boltzmann constant and $T$ is the system temperature. The weight function for dissipative force and random force are $w_{\mathrm{R}}\left(\mathrm{r}_{\mathrm{ij}}\right)=1-r_{\mathrm{ij}} / r_{\mathrm{C}}$, and $w_{\mathrm{D}}\left(\mathrm{r}_{\mathrm{ij}}\right)=\left(1-r_{\mathrm{ij}} / r_{\mathrm{C}}\right)^{2}$, respectively ${ }^{38}$.

\subsection{Fluid properties and validation}

In this section, we test the MDPD method by comparing our simulation results of a droplet spreading on a solid surface with the corresponding experimental results in Ref. 39 Generally, MDPD operates in reduced units so that $k_{\mathrm{B}} T=1.0$ and $r_{\mathrm{C}}=1.0$. A common choice for the interaction parameters between liquid particles then is $A_{11}=-40, B_{11}=25$ and $r_{\mathrm{d}}=0.75^{13,28,37}$.

Figure 2a shows the density profile of a spherical droplet with the density of the droplet $\rho=$ 6.09, which is slightly different from $\rho=6.0$ as obtained in Ref. 28 with exactly the same set of parameters. Surface tension is an important interface property in the interaction between a liquid droplet and a solid surface. Conducting a simulation of a thin liquid film, the surface tension can be derived by subtracting the mean tangential stress components (i.e. $P_{\mathrm{xx}}$ and $P_{\mathrm{yy}}$ ) from the 
normal (i.e. $\left.P_{\mathrm{zz}}\right): \sigma=\int_{-\infty}^{\infty} d z\left[P_{z z}-\frac{1}{2}\left(P_{x x}+P_{y y}\right)\right]^{40}$. The calculated surface tension is $\sigma=7.51$, which matches the value in Ref. $28(\sigma=7.51 \pm 0.04)$.

The viscosity of the liquid can be assessed separately from $\rho$ and $\sigma$ and directly obtained by the periodic Poiseuille flow method ${ }^{37,41,42}$. In this validation, the parameter $\gamma$ equals 0.005 . A computational box filled with 4677 particles is divided into two symmetric regions and a body force along the $z$ direction is applied to each particle located in a half of the box, while an equal force in the opposite direction is applied to particles in the other half. This distribution of forces leads to parabolic velocity profiles on either side of the box, as seen in Figure 2b. Fitting the simulated time-averaged velocity in $z$-direction as a function of $x$ with a quadratic function - as shown in Figure $2 \mathrm{~b}$ - then provides us with an estimate for the kinematic viscosity of $v=0.40$.

The relationship between the MDPD units of length $\left(L_{\mathrm{MDPD}}\right)$, mass $\left(M_{\mathrm{MDPD}}\right)$ and time $\left(T_{\mathrm{MDPD}}\right)$ can be mapped to specific properties of real liquids (i.e. density $\rho^{*}$, surface tension $\sigma^{*}$ and viscosity $v^{*}$ ) according to Eq. (4). ${ }^{37}$

$$
\begin{aligned}
& M_{\mathrm{MDPD}}=L_{\mathrm{MDPD}}^{3} \frac{\rho^{*}}{\rho} \\
& T_{\mathrm{MDPD}}=\left(M_{\mathrm{MDPD}} \frac{\sigma}{\sigma^{*}}\right)^{0.5} \\
& \frac{L_{\mathrm{MDPD}}^{2}}{T_{\mathrm{MDPD}}}=\frac{v^{*}}{v}
\end{aligned}
$$

As an example, the ethylene glycol solution as used in Ref. $39\left(\rho^{*}=1056 \mathrm{~kg} / \mathrm{m}^{3}, \sigma^{*}=56.5 \mathrm{mN} / \mathrm{m}\right.$ and $v^{*}=3.03 \times 10^{-6} \mathrm{~m}^{2} / \mathrm{s}$ ) can be matched by $\rho=6.09, \sigma=7.51$ and $v=0.4$, respectively. So we determine the scaling coefficients between the physical and MDPD units as $L_{\mathrm{MDPD}}=1.32 \times 10^{-6} \mathrm{~m}$, $M_{\mathrm{MDPD}}=4.01 \times 10^{-16} \mathrm{~kg}$, and $T_{\mathrm{MDPD}}=2.31 \times 10^{-7} \mathrm{~s}$ by Eq. (4).

Droplets with different static contact angle $\left(\theta_{\mathrm{c}}\right)$ on the substrate can be created by altering the 
solid-liquid interaction parameter $A_{\mathrm{sl}}$. The relationship between $A_{\mathrm{sl}}$ and $\theta_{\mathrm{c}}$ is shown in Figure $2 \mathrm{c}$. The range of $A_{\mathrm{sl}}$ is from -37 to -4 and the range of $\theta_{\mathrm{c}}$ is from $45^{\circ}$ to $180^{\circ}$. The contact angle is determined by fitting a circle to a cross section of the gas-liquid interface which is defined as the surface that has a density approximately half of the bulk density of the droplet. The simulated results in Figure 2c agree well with the values in Ref. 43.

For further validation, we now consider the case of an impacting droplet for which experimental data is available (Ref. 39). The time evolution of the impacting droplet with $W e=$ 12.1 in terms of its dimensionless height $H^{*}=H / D_{0}$ and spreading factor $D^{*}=D / D_{0}$ with $D_{0}$ the initial droplet diameter is shown in Figure 3. The maximum spreading factor $\left(D_{\max }^{*}\right)$ and the spreading time (from the impact to the maximum spread) are 1.57 and $9.2 \mu \mathrm{s}$,corresponding to the value $1.4 \pm 0.2$ and $9.2 \pm 2.2 \mu \mathrm{s}$ in the reference ${ }^{39}$. Finally, the droplet reaches a stable state and the contact angle reaches $90^{\circ}$. It should be noted that we consider the surfaces as ideal. The presence of contact angle hysteresis on real surfaces can lead to a retraction of the droplet in an experiment ${ }^{24,44}$. After the comparison between the simulation and experimental values, we feel confident to use the MDPD method to predict droplet spreading on heterogeneous substrates.

\subsection{Problem specifications}

The schematic for a droplet deposition on the periodically stripe-patterned substrate is shown in Figure 4. Here the hydrophobic stripe width equals to the hydrophilic stripe width, represented by $W_{\mathrm{s}}$. The ratio of stripe width $W_{\mathrm{s}}$ over initial droplet diameter $D_{0}$ is defined as $\beta$. It is in the range 0.5 to 1.0 , so that in this work the droplet is in the Beyond-Cassie-Baxter regime ${ }^{18}$. First we ignore the inertial effect and study the droplet wetting behavior as the droplet deposits starting from three different positions (the middle of the hydrophobic stripe - Case1; the middle 
of the hydrophilic stripe - Case2; and exactly the border between two different stripes - Case3), seen as Figure 4a-c. Then we choose Case 3 as an example to study inertial droplet impingement and splitting behavior on the single-striped surface and multi-striped surface.

A computational domain of size $160 \times 100 \times 60$ is adopted in the three-dimensional simulation with periodic boundary condition in the $x$ and $y$ directions. The $x$ direction is normal to the stripes and $y$ direction is in the direction of the stripes. The $z$ direction is the direction normal to the substrate. Each droplet has diameter $D_{0}=28$ and consists of 69849 MDPD particles $(\rho=6.09, \sigma=7.51$ and $v=0.80(\gamma=1))$, and the droplet matches the liquid with $\rho^{*}=1056 \mathrm{~kg} / \mathrm{m}^{3}$, $\sigma^{*}=56.5 \mathrm{mN} / \mathrm{m}, v^{*}=6.03 \times 10^{-6} \mathrm{~m}^{2} / \mathrm{s}$, and its diameter equals to $37 \mu \mathrm{m}$. The substrate contains between 156294 and 310182 frozen MDPD particles. The bounce forward boundary condition ${ }^{45}$ is applied to prevent liquid particles from penetrating the wall. Gravity is neglected. A standard velocity-Verlet algorithm is applied for the simulations, using the value of 0.5 for the empirical parameter $^{11}$. The time step was set to 0.01 in MDPD units.

\section{Results and discussion}

\subsection{Droplet wetting behavior without inertia}

\subsubsection{The effect of deposition position}

It has been reported that the droplet shape in spreading is sensitive to the deposition position when the droplet radius is of the same order of magnitude as the stripe width ${ }^{18}$. Here, we deposit the droplet in three different positions by placing it close to the surface (within $1.0 r_{C}$ ) with zero velocity so that the droplet spreads on the surface due to the attractive interaction between liquid and solid surface. The intrinsic contact angle of the hydrophilic stripe $\theta_{\text {lic }}$ and hydrophobic stripe $\theta_{\text {bic }}$ are $45^{\circ}$ and $115^{\circ}$, respectively. The relative hydrophobicity $\alpha$ is 1 and $\beta$ is 0.5 . 
Figure 5 shows the droplet wetting process in terms of time evolutions; snapshots are shown in Figure 6. Dimensionless scales $W^{*}$ and $L^{*}$ are introduced to describe the droplet morphology, defined as the width and the length of droplet divided by the droplet initial diameter $D_{0}$, respectively. At the beginning of the wetting process, until $W^{*}$ reaches 0.5 , the droplet spreads isotropically with the same spreading rate in Case 1 and Case 2 (Figure 6,t=10) since the droplet is spreading on a homogeneous part of the surface. At the moment when reaching $W^{*}=0.5$, the droplet in Case 1 reaches the hydrophilic part of the surface and spreads faster in the $x$-direction as compared to the $y$-direction. The opposite occurs in Case 2 where the droplet has a preference to stay on the hydrophilic stripe it was deposited on. Case 2 also has a recoiling stage with the droplet retracing in the $x$-direction after $t \approx 100$ so as to reach a larger contact angle $\theta_{\perp}$ normal to the stripes. Case 3 shows a migration of the droplet towards a hydrophilic stripe (see Figure 6c) and then evolves in a way similar to Case 2 (compare Figure $5 \mathrm{~b}$ and $5 \mathrm{c}$ ).

The energy barrier in perpendicular direction to the stripe causes the droplet spreading along the stripe and a lozenge shape droplet forms in Cases 2 and 3 (Figure $6 \mathrm{~b} \& \mathrm{c}, t=500$ ). Differently, a butterfly-like droplet with a width equal to 1.5 times its initial diameter forms in Case 1 (Figure 6a, $t=500$ ), which also has been observed in experiments ${ }^{18}$. Interestingly, the droplet length and width are almost unchanged if the wettability of the hydrophobic stripe changes in Case 1 (inset in Figure 5a). The length of the neck, however, is sensitive to the wettability of the hydrophobic stripe.

\subsubsection{The effect of the wettability of the hydrophobic stripes}

We now focus on the equilibrium shape of a droplet that is released - still without inertia - on the border between a hydrophobic and hydrophilic stripe. As shown in Figure 6 (Case 3), the droplet will migrate to the hydrophilic stripe. We study the effects of the droplet size relative to the stripe width ( $\beta$ in the range 1.0 to 0.5 ) and the wettability of the hydrophobic stripes with $\theta_{\text {bic }}$ in the range $93^{\circ}$ to $180^{\circ}$, while keeping fixed $\theta_{\text {lic }}=45^{\circ}$. The variation in $\theta_{\text {bic }}$ is achieved by 
changing $A_{\text {bic }}$. Results of the simulations have been summarized in terms of the parallel and perpendicular contact angles $\left(\theta_{\|}\right.$and $\theta_{\perp}$, respectively) in Figure 7 , and in terms of the droplet's aspect ratio in Figure 8.

In all cases, the footprint of the droplet extended over the entire width of the hydrophilic stripe, while not covering the hydrophobic stripe, see Figure $7 \mathrm{~b}$ for two typical droplet shapes. The largest droplets - relative to the stripe width - are the ones with $\beta=0.5$. As shown in Figure $7 \mathrm{a}$, with increasing hydrophobicity initially their $\theta_{\perp}$ closely follows $\theta_{\text {bic }}$ and then levels off to $\theta_{\perp}$ $\approx 135^{\circ}$. For smaller droplets, the dependency of $\theta_{\perp}$ with $A_{\text {bic }}$ is much weaker. If $\beta \geq 0.7, \theta_{\perp}$ hardly depends on the hydrophobicity. Given that $\theta_{\|}$is formed on the hydrophilic stripe, it matches closely with $\theta_{\text {lic }}$ that was set at $45^{\circ}$. Slight variations in $\theta_{\|}$are due to uncertainties in estimating this angle. Given the shape of the droplet in the parallel direction we cannot estimate this angle via a circle fit. Therefore, we extract a part of the boundary points near the surface (up to 0.3 times the initial drop diameter above the surface) and connect those points with a polynomial curve of fourth order (red curves in Fig. 7c). The contact angle is derived from the slope of the polynomial at the surface.

Figure 8 shows the aspect ratio of equilibrium droplet as a function of $\beta$ and $\theta_{\text {bic. }}$ As we saw in Figure $7 \mathrm{a}$, the shape of the droplet - now in terms of its aspect ratio - only depends on $\beta$ for sufficiently high hydrophobicity, i.e. for $\theta_{\mathrm{bic}} \geq 155^{\circ}$. It is interesting to note that for lower $\theta_{\text {bic }}$ the aspect ratio as a function of $\beta$ goes through a maximum.

\subsection{Droplet impingement behavior considering inertial effect}

\subsubsection{Droplet impingement on single stripe surface}

In this section, we consider the effect of droplet inertia on droplet morphology. In Song et al.'s ${ }^{23}$ experiment, a hydrophilic surface coating with a slim superhydrophobic stripe was found performing well in splitting droplet if the Weber number (We) of the droplet was sufficiently 
high, indicating the potential of this chemically striped surface for splitting droplets in microfluidics applications. Here we investigated the droplet splitting behavior on the hydrophilic surface coated with a superhydrophobic stripe with various widths. Compared with a low $\beta$ in Song et al.'s experiment ${ }^{23}(\beta \approx 0.2), \beta$ is larger in this work with the range of 0.5 to 1.0 . The contact angles of the hydrophilic surface and the hydrophobic stripe are $45^{\circ}$ and $170^{\circ}$, respectively.

Taking the case $\beta=0.5$ as an example, we investigate the effect of droplet deposition position on the volume fraction $\left(V_{\mathrm{f}}\right)$ and maximum-footprint-area fraction $\left(A_{\mathrm{f}}\right)$ of split droplets, and the results are shown in Figure 9. Note that $V_{\mathrm{f}}$ is obtained by the split droplet volume scaled to the initial droplet volume, and $A_{\mathrm{f}}$ is obtained by each part of maximum-footprint-area scaled to the total footprint-area on the hydrophilic surface. In Figure 9 , we can see that $V_{\mathrm{f}}$ matches $A_{\mathrm{f}}$, and this characteristic agrees well with that proposed by Song et al. ${ }^{23}$. It can also be seen in Figure 9 that the difference of volume fraction between two split droplets increases approximately linearly with $L / D_{0}$ until the entire droplet ends up on one side of the stripe and no split has happened.

Taking Case 3 (as defined in Figure. 4) as an example, we illustrate the asymmetric splitting behavior on a single stripe surface with $\beta$ equal to 0.5 and 0.9 at $W e=22.7$ in Figure 10 . The droplet exhibits two equilibrium states: split off or shift to the hydrophilic area. During the impingement, the projection of the droplet remains circular as it radially spreads outwards (for $t$ $\leq 10$ ), indicating that the droplet's inertia dominates the spreading process during this stage. Subsequently, the droplets behave differently due the different values of $\beta$. In the case $\beta=0.5$, the droplet inertia (characterized by the Weber number) is high enough for the droplet to cross the superhydrophobic stripe and enter the hydrophilic area right of the stripe $(t=10)$. After reaching its maximum spreading on the superhydrophobic stripe $(t=30)$, the part of the droplet on the superhydrophobic stripe starts to get squeezed, and a liquid neck forms. From then on, the 
droplet keeps stretching along the direction perpendicular to the stripe. The liquid neck collapses and the droplet splits into two (at $t=230$ ). In the case $\beta=0.9$, the edge of the droplet fails to reach the hydrophilic area at the opposite side of the stripe because its inertia and associated kinetic energy is insufficient for this. After reaching the maximum spreading $(t=30)$, the edge of the droplet on the superhydrophobic stripe retracts while the liquid on the hydrophilic area still spreads outwards and the droplet becomes shell-like with a gibbous frontier on the edge (in the side view when $t=40$ ). Gradually, the part of the droplet on the superhydrophobic stripe retracts back to the hydrophilic area and stays in hydrophilic area at its equilibrium state $(t \geq$ 230).

The eventual droplet state is co-determined by droplet inertia and surface properties, which can be characterized by $W e$ and $\beta$ as well as the contact angles of the hydrophilic surface and the hydrophobic stripe. Here we focus on the effects of $W e$ and $\beta$ at fixed $\theta_{\mathrm{bic}}=170^{\circ} \theta_{\mathrm{lic}}=45^{\circ}$. In the Appendix we show the (limited) sensitivity of these contact angles on the final droplet morphology.

The effect of $W e$ and $\beta$ on the droplet equilibrium shape is visualized in Figure 11a, where the red circle represent droplet split off cases. To find the critical boundary for droplet split-off, we did additional simulations of droplet impingent on a homogeneous superhydrophobic surface over a range of We with a fixed superhydrophobic contact angle of $170^{\circ}$. For convenience, we introduce the maximum spreading radius factor, $R_{\max }^{*}$, defined as the maximum value of $R^{*}$ (the ratio between droplet spreading radius and $D_{0}$ ). The history of $R^{*}$ is shown in Figure $11 \mathrm{c}$. We find that initially $R^{*}$ increases with time and then decreases after reaching the peak. Given that mostly - the peak in radius is not very sharp, we take an average over a few data points around the peak values to determine $R_{\max }^{*}$ as indicated in Figure 11c. The relationship between $R_{\max }^{*}$ and $W e$ is plotted in Figure 11a \& b, as black squares. 
Coming back to droplet deposition on a single-stripe surface, at a specific Weber number, the droplet splits if $R_{\max }^{*}>\beta$, whereas otherwise it will shift to the hydrophilic side. It implies that the hydrophilic surface with a superhydrophobic stripe has the capability to split the droplet as long as the droplet can get across the stripe at its maximum spreading.

Therefore, the concept of $R_{\max }^{*}$ can help us to predict the result of droplet impingement: splitting off or a shift to hydrophilic side. Power law behavior of $R_{\max }^{*} \propto W e^{\alpha_{1}}$ has been proposed based on theoretical analysis. ${ }^{46}$ A scaling law of $R_{\max }^{*} \propto W e^{0.5}$ has been found by a balance between inertia and surface tension forces when We is large enough and viscous energy dissipation is neglected ${ }^{47,48}$. On the other hand, Clanet et al. ${ }^{49}$ regarded the maximum droplet shape as a balance between gravity and surface forces. They determined the droplet thickness at maximum spread based on capillary length and obtained $R_{\max }^{*} \propto W e^{0.25}$ by imposing volume conservation. Recently, Lin et al. ${ }^{46}$ found the above exponents in scaling law also related to the viscosity of the droplet in their experiments, where $\alpha_{1}$ can be as high as 0.25 for water, and as low as 0.1 for a $397.8 \mathrm{mPa} \cdot \mathrm{s}$ viscous liquid. Based on our simulations, we found $R_{\max }^{*} \propto W e^{0.20}$, see Figure $11 b$.

\subsubsection{Droplet impingement on periodically hydrophilic and hydrophobic stripes}

Now, we investigate droplet splitting behavior on a periodic multi-striped surface. The deposition point and the contact angles are unchanged. The value of We has been fixed at 22.7, so that the droplet splits when $\beta \leq 0.75$ on a single-striped surface, as shown in Figure 11 . However, we find the droplet exhibits a "butterfly" shape on multi-striped surface if $\beta=0.5$ (Figure 12a, $t=675$ ), indicating the influence of the multi-striped mode on the eventual droplet state.

Figure 12 shows more details about an impinging droplet on multi-striped surfaces with various $\beta$. Initially $(t \leq 10)$, the droplet sets in the spreading stage, where the droplet inertia 
dominates the evolution process so that the droplet spreads outwards with the same speed in all three cases. Subsequently, the droplet behaves differently for different $\beta$. For $\beta=0.5$, the liquid on the superhydrophobic stripes retracts when $t \geq 30$. During this retracting process, the gibbous part of the droplet on hydrophobic stripes prefers to move directly to the hydrophilic stripes, while the liquid on the right hydrophilic stripe is still stretching outwards until it fills up the entire width of the hydrophilic stripe (Figure.12a, $\beta=0.5, t=100$ ). The droplet neck forms during droplet recoiling. However, compared to the spreading behavior on a single-striped surface, more liquid is contained in the droplet neck on a multi-striped surface. This results in the deceleration of the neck-shrink, as illustrated in Figure 12a. Finally, the droplet remains intact after impingement and becomes "butterfly" shape.

For cases with larger $\beta$, there is enough space on the right hydrophilic stripe for liquid to stretch and spread radially, generating the collapse of the neck and finally droplet split-off (Figure $12 \mathrm{~b}, \beta=0.7, t=175$ ). In addition, the periodic chemical stripes reshape the split droplet to an elongated shape. If the stripe is wide enough so as to hinder the droplet stepping over the superhydrophobic stripe, the droplet will vibrate on the hydrophilic stripe until it consumes the remaining energy. Finally, the droplet exhibits an elongated shape without any splitting, as revealed in Figure 12c.

The aforementioned droplet behavior on a multi-striped surface can be quantitatively described by analyzing the energy associated to the droplet. The conversion of energy during the process of droplet impingement on the substrate can be expressed as

$$
E_{k}^{0}+E_{s}^{0}+A_{s v} \sigma_{s v}=A_{l v} \sigma_{l v}+A_{l s} \sigma_{l s}+\left(A_{s v}-A_{l s}\right) \sigma_{s v}+E_{k}+E_{v},
$$

where, $E_{k}^{0}$ and $E_{s}^{0}$ denote the droplet initial kinetic energy and surface energy, respectively. $A_{\mathrm{sv}}, A_{\mathrm{lv}}$ and $A_{\mathrm{ls}}$ represent the area of solid-vapor surface, liquid-vapor surface and liquid-solid surface, and their corresponding surface tension are $\sigma_{\mathrm{sv}}, \sigma_{\mathrm{lv}}$, and $\sigma_{\mathrm{ls}}$. Clearly, the interfacial 
energy of solid-vapor surface can be removed on the both side of the equation. Therefore, we have:

$$
E_{s}^{0}+E_{k}^{0}=E_{s}+E_{k}+E_{v}
$$

where

$$
E_{s}=A_{l v} \sigma_{l v}+A_{l s}\left(\sigma_{l s}-\sigma_{s v}\right)
$$

From the Young's equation we know that $\sigma_{l s}-\sigma_{s v}=-\sigma_{l v} \cos \theta_{c}$. Hence,

$$
E_{s}=A_{l v} \sigma_{l v}-A_{l s, \text { bic }} \sigma_{l v} \cos \theta_{b i c}-A_{l s, \text { lic }} \sigma_{l v} \cos \theta_{l i c}
$$

where $A_{\mathrm{ls}, \mathrm{bic}}$ and $A_{\mathrm{ls} \text {,ic }}$ are the area of liquid-solid surface on the hydrophobic stripes and hydrophilic stripes, respectively.

$E_{\mathrm{k}}$ can be calculated by

$$
\begin{aligned}
& \overrightarrow{\mathrm{v}}_{\text {mean }}=\frac{1}{N} \sum_{i=1}^{N} \overrightarrow{\mathrm{v}}_{i} \\
& E_{\mathrm{k}}=\frac{1}{2} M\left|\overrightarrow{\mathrm{v}}_{\text {mean }}\right|^{2}=\frac{1}{2} M\left(v_{\mathrm{x}, \text { mean }}^{2}+v_{\mathrm{y}, \text { mean }}^{2}+v_{\mathrm{z}, \text { mean }}^{2}\right)
\end{aligned}
$$

where $v_{\mathrm{x}}, v_{\mathrm{y}}, v_{\mathrm{z}}$ are the velocity components of each particle and $M$ is the droplet mass, i.e. the number of liquid particles since each particle mass equals to 1 . The normalized kinetic energy $\left(E_{k}^{*}\right)$ and surface energy $\left(E_{s}^{*}\right)$ are obtained by $E_{k} / E_{s}^{0}$ and $E_{s} / E_{s}^{0}$, respectively. The viscous dissipation energy $E_{\mathrm{v}}$ is related to the droplet viscosity and velocity.

Figure 13 shows the time history of the scaled energy $E_{k}^{*}$ and $E_{s}^{*}$. At the beginning of deposition $(t \leq 10)$, the spreading droplet has a nearly spherical cap shape. The loss rate of $E_{k}^{*}$ is more than the loss rate of $E_{s}^{*}$. Then the droplet starts spreading asymmetrically, where $E_{k}^{*}$ still decreases while $E_{s}^{*}$ rises slightly. We note that $E_{s}^{*}$ reaches its local maximum when $E_{k}^{*}$ reduces to zero (at $t=30)$, and there $E_{s}^{*}(\beta=0.9) \approx E_{s}^{*}(\beta=0.7)>E_{s}^{*}(\beta=0.5)$. However, the droplet does not come to a halt; surface energy starts to convert into kinetic energy. The droplet now is in an energy fluctuation stage. The fluctuation of $E_{s}^{*}$ and $E_{k}^{*}$ is gentle for $\beta=0.5$ and 
more violent for $\beta=0.9$ before the droplet reaches equilibrium. The fluctuations relate to the morphologic changes in Figure 12. Finally, the droplet comes to rest when $t>500$. In addition, $E_{s}^{*}$ and $E_{k}^{*}$ are stable. We can see that $E_{s}^{*}(\beta=0.5)>E_{s}^{*}(\beta=0.7)>E_{s}^{*}(\beta=0.9)$ in steady state, indicating that smaller $\beta$ helps forming droplets with higher surface energy.

Figure 14a illustrates that $W e$ and $\beta$ affect the eventual droplet shape simultaneously. Clearly, small We and large $\beta$ tend to make the droplet elongated on the stripe. This is because lower kinetic energy cannot sufficiently help the droplet overcome the energy barrier formed by the superhydrophobic stripes, which results in the droplet retracting and spreading along the hydrophilic stripe. The equilibrium shape of the droplet generally changes from elongated to split as We increases. The critical boundary, expressed by $R_{\max }^{*}$ in single-striped cases, is also valid for multi-striped cases. Generally, droplets split if $R_{\max }^{*}<\beta$. However, the "butterfly" shape occurs if $\beta \leq 0.6$. In addition, there is a mixed regime where elongated or split droplets occur randomly. This is when $\beta$ is near the maximum spreading radius factor $R_{\max }^{*}$ (dotted line in Figure 14a).

Figure 14b shows $E_{s}^{*}$ as a function of $W e$ for two different $\beta$ cases. Clearly, the droplet transforms from low surface energy (elongated shape) to high energy (split-off or even "butterfly" shape) as $W e$ increases. The value of $E_{s}^{*}$ remains constant when the droplet is elongated or has a "butterfly" shape. For the "butterfly" shape, there is little difference in $E_{s}^{*}$ between the two $\beta$ cases. For the elongated shape, however, $E_{s}^{*}$ is much lower in the higher $\beta$ case, which means more energy is required to transform the droplet state from elongated to butterfly for higher $\beta$.

The chemically multi-striped surface is found to transform the droplet into three morphologies when droplet inertia is considered. The diagram for the droplet morphology may be used in microfluidic system to control droplet shape or split the droplet. It should be noted that the droplet morphology only depends on $\beta$ and not on We if the droplet is elongated or "butterfly" 
shaped since the surface energy stays constant, as shown in Figure 14b. When the droplet is split, one can get a desired split by adjusting $\beta$ and $W e$.

As stated above, the results presented in this section are for $\theta_{\mathrm{bic}}=170^{\circ}$ and $\theta_{\mathrm{lic}}=45^{\circ}$. In the Appendix the sensitivity of the contact angles has been investigated. Additionally, it should be emphasized that the surfaces in our simulation are smooth since we focus on the effect of contact angle difference between superhydrophobic and hydrophilic stripe on droplet morphology. However, experiment is surface roughness, especially for superhydrophobic surfaces that are usually decorated by micro-/nanostructures.

\section{Conclusions}

In this work, three-dimensional many-body dissipative particle dynamics (MDPD) simulation was employed to investigate the droplet impingement and subsequent wetting behavior on chemically stripe-patterned surfaces with $\beta$ (which is the ratio of stripe width over droplet diameter) ranging from 0.5 to 1.0. Two scenarios, with and without inertial effects, were explored.

To validate the MDPD method in describing the droplet impingement behavior on solid surfaces, we firstly matched the physical properties of a given liquid with the dimensionless MDPD system. Then comparison between the simulation and experimental results showed the ability of MDPD in properly simulating droplet impingement behavior.

Subsequently, we investigated the droplet wetting behavior without inertial effects on striped surfaces. We found that a recoiling stage was only present when the droplet deposited on the middle of a hydrophilic stripe or on the border of the stripe. The latter case was studied more extensively. We found that the parallel contact angle was constant and was close to the intrinsic contact angle on the hydrophilic stripes. On the other hand, when the hydrophobic stripes are superhydrophobic, the perpendicular contact angle and aspect ratio of the droplet only depend on $\beta$. 
As for the case with inertial effects, droplet impingement behavior on single-striped surfaces and on multi-striped surfaces were both investigated, where the contact angle $\theta_{\text {bic }}$ is larger than $150^{\circ}$. For single-striped surfaces, the droplet splits into two with the distribution of volume approximately equal to the maximum-footprint-area distribution. The Weber number (We) affects the droplet morphology and the critical condition for droplet split-off is identified as $R_{\max }^{*}=0.41 W e^{0.20}$ where the droplet splits when $R_{\max }^{*}>\beta$. Different from impinging on single-striped surfaces, three droplet morphologies, elongated, split off and "butterfly" shape, were observed on multi-striped surfaces. Both $W e$ and $\beta$ affect the droplet morphology. A droplet splits for high We and low $\beta$, while "butterfly" droplet occurs if $\beta$ is less than or equal to 0.6. The critical condition $R_{\max }^{*}=0.41 W e^{0.20}$ is also valid to predict the droplet morphology on multi-striped surfaces with elongated droplets if $R_{\max }^{*}<\beta$. It was also shown - see the Appendix - that this criterion is not sensitive to the specific values of $\theta_{\text {bic }}$ and $\theta_{\text {lic }}$.

Finally, through the analysis of the scaled surface energy of the droplet, we found that $\beta$ is the only factor affecting the shape of elongated and "butterfly" droplets. Overall, the current study provides the potential of precise control of droplet morphology in the Beyond-Cassie-Baxter regime by modifying the parameter $\beta$, stripe wettability and We. It could help support the design of microfluidic devices where the droplet morphology needs careful control.

Non-Newtonian liquid droplets are widely used in the applications such as inkjet printing ${ }^{50}$ and bioprinting ${ }^{51}$. Different from Newtonian fluid, the viscosity of non-Newtonian fluid is strongly dependent on the applied stress. Considering the special behavior of non-Newtonian fluid is therefore of practical as well as of academic interest. We will be working on the spreading of such liquid droplet in the Beyond-Cassie-Baxter regime in future research.

\section{Acknowledgement}

The authors gratefully acknowledge the financial support from the National Key R\&D Program of China (2017YFB0306701), National Natural Science Foundation of China 
(No.21676007), the Fundamental Research Funds for the Central Universities (XK1802-1), and China Scholarship Council.

\section{Appendix}

\section{Sensitivity analysis of the intrinsic contact angles}

The critical condition $R_{\max }^{*}=0.41 \mathrm{We} e^{0.20}$ is derived from the case $\theta_{\text {bic }}=170^{\circ} \theta_{\text {lic }}=45^{\circ}$. Here we estimate the validity of this expression for more general situations by changing the intrinsic contact angles on the hydrophobic and hydrophilic parts of the substrate.

Firstly, the contact angle on hydrophobic stripes is changed from $170^{\circ}$ to $155^{\circ}$ while the hydrophilic stripes wettability remained unchanged. Before checking the droplet morphology on this new multi-striped surface $\left(\theta_{b i c}=155^{\circ} \theta_{\text {lic }}=45^{\circ}\right)$, we study droplet impingement behavior on homogeneous surface. Figure 15 shows the $R_{\max }^{*}$ vs. We for Case $\theta_{\mathrm{c}}=155^{\circ}$ and Case $\theta_{\mathrm{c}}=170^{\circ}$. If We $<35, R_{\max }^{*}$ is larger in case $\theta_{\mathrm{c}}=155^{\circ}$ than in case $\theta_{\mathrm{c}}=170^{\circ}$. When $W e>35$, droplet inertia dominates the impact process and there is hardly any difference in $R_{\max }^{*}$. We can also see that the expression $R_{\max }^{*}=0.41 W e^{0.20}$ is valid for the Case $\theta_{\mathrm{c}}=155^{\circ}$. Therefore, we still use this relationship as the criterion for this new multi-striped surface $\left(\theta_{b i c}=155^{\circ} \theta_{l i c}=45^{\circ}\right)$.

Now, we study the droplet morphology on the new multi-striped surface $\left(\theta_{b i c}=155^{\circ} \theta_{\text {lic }}=45^{\circ}\right)$ and the results are shown in Figure 16b. Comparing Figure 16a and Figure16b, we can see that the criterion $R_{\max }^{*}<\beta$ is fundamentally valid for cases that have $\theta_{b i c}=155^{\circ} \theta_{\text {lic }}=45^{\circ}$. However, more "butterfly" droplets can be found for $\theta_{b i c}=155^{\circ} \theta_{\text {lic }}=45^{\circ}$. In addition, the droplet splits when $\beta=0.5$ in case $\theta_{b i c}=155^{\circ} \theta_{\text {lic }}=45^{\circ}$ but not if $\theta_{b i c}=170^{\circ} \theta_{\text {lic }}=45^{\circ}$.

Next, we increase the intrinsic contact angle on hydrophilic stripes from $45^{\circ}$ to $60^{\circ}$ and fix the hydrophobic stripes wettability to again study the droplet morphology on this multi-striped surface $\left(\theta_{b i c}=170^{\circ}, \theta_{\text {lic }}=60^{\circ}\right)$. Results are shown in Figure 16c. Undoubtedly, the criterion $R_{\max }^{*}<\beta$ is valid in Figure 16c, since the hydrophobic stripes wettability unchanged. The 
number of "butterfly" shape marked in green in Figure 16c is less than that in Figure 16a, indicating that it need higher We for droplet to generate "butterfly" shape in larger $\theta_{\text {bic }}$ cases, compare to smaller $\theta_{\text {bic }}$ cases.

To sum up, there is hardly any sensitivity with respect to the wettability of the hydrophobic and hydrophilic parts of the substrate, indicating the above-mentioned criterion can be used generally.

\section{Reference}

1. Chen Y, He B, Lee J, Patankar Neelesh A. Anisotropy in the wetting of rough surfaces. Journal of Colloid and Interface Science. 2005;281:458-464

2. Semprebon C, Mistura G, Orlandini E, Bissacco G, Segato A, Yeomans JM. Anisotropy of Water Droplets on Single Rectangular Posts. Langmuir. 2009;25:5619-5625.

3. Yao X, Song Y, Jiang L. Applications of bio-inspired special wettable surfaces. Advanced materials. 2011;23:719-734.

4. Wu L, Dong Z, Kuang M, et al. Printing patterned fine 3D structures by manipulating the three phase contact line. Advanced Functional Materials. 2015;25:2237-2242.

5. Shang X, Luo Z, Bai B. Droplets trapped by a wetting surface with chemical defects in shear flows. Chemical Engineering Science. 2019;195:433-441.

6. Jansen HP, Zandvliet HJW, Kooij ES. Evaporation of elongated droplets on chemically stripe-patterned surfaces. International Journal of Heat and Mass Transfer. 2015;82:537-544.

7. Macis M, Lugli F, Zerbetto F. Modeling living cells response to surface tension and chemical patterns. ACS Applied Materials \& Interfaces. 2017;9:19552-19561.

8. Patrick Jansen H, Sotthewes K, Zandvliet HJW, Kooij ES. Potential of lattice Boltzmann to model droplets on chemically stripe-patterned substrates. Applied Surface Science. 2016;361:122-132. 
9. Wu Y, Wang F, Selzer M, Nestler B. Investigation of equilibrium droplet shapes on chemically striped patterned surfaces using phase-field method. Langmuir. 2019;35:8500-8516.

10. Zou L, Wang H, Zhu X, Chen R, Ding Y, Liao Q. Maximum spread of droplets on chemically striped surfaces. AIChE Journal. 2019:e16774.

11. Varagnolo S, Schiocchet V, Ferraro D, et al. Tuning drop motion by chemical patterning of surfaces. Langmuir. 2014;30:2401-2409.

12. Carmeliet J, Chen L, Kang Q, Derome D. Beyond-Cassie mode of wetting and local contact angles of droplets on checkboard-patterned surfaces. Langmuir. 2017;33:6192-6200.

13. Lin $\mathrm{C}$, Chen $\mathrm{S}$, Xiao L, Liu Y. Tuning drop motion by chemical chessboard-patterned surfaces: a many-body dissipative particle dynamics study. Langmuir. 2018;34:2708-2715.

14. Bliznyuk O, Vereshchagina E, Kooij ES, Poelsema B. Scaling of anisotropic droplet shapes on chemically stripe-patterned surfaces. Physical review. E, Statistical, nonlinear, and soft matter physics. 2009;79:041601.

15. Damle VG, Rykaczewski K. Nano-striped chemically anisotropic surfaces have near isotropic wettability. Applied Physics Letters. 2017;110:171603.

16. Bliznyuk O, Jansen HP, Kooij ES, Poelsema B. Initial spreading kinetics of high-viscosity droplets on anisotropic surfaces. Langmuir. 2010;26:6328-6334.

17. Jansen HP, Bliznyuk O, Kooij ES, Poelsema B, Zandvliet HJW. Simulating anisotropic droplet shapes on chemically striped patterned surfaces. Langmuir. 2011;28:499-505.

18. Wang Y, Jian M, Liu H, Zhang X. Anisotropic Wetting of droplets on stripe-patterned chemically heterogeneous surfaces: Effect of length ratio and deposition position. Langmuir. 2018. 
19. Jansen HP, Sotthewes K, Ganser C, Zandvliet HJW, Teichert C, Kooij ES. Shape of picoliter droplets on chemically striped patterned substrates. Langmuir. 2014;30:11574-11581.

20. Léopoldès J, Dupuis A, Bucknall DG, Yeomans JM. Jetting micron-scale droplets onto chemically heterogeneous surfaces. Langmuir. 2003;19:9818-9822.

21. Brinkmann M, Lipowsky R. Wetting morphologies on substrates with striped surface domains. Journal of Applied Physics. 2002;92:4296-4306.

22. Lipowsky R, Brinkmann M, Dimova R, Franke T, Kierfeld J, Zhang X. Droplets, bubbles, and vesicles at chemically structured surfaces. Journal of Physics: Condensed Matter. 2005;17:S537-S558.

23. Song D, Song B, Hu H, Du X, Zhou F. Selectively splitting a droplet using superhydrophobic stripes on hydrophilic surfaces. Physical Chemistry Chemical Physics. 2015;17:13800-13803.

24. Zhao J, Chen S, Liu Y. Dynamical behaviors of droplet impingement and spreading on chemically heterogeneous surfaces. Applied Surface Science. 2017;400:515-523.

25. Jansen HP, Sotthewes K, Ganser C, Teichert C, Zandvliet HJW, Kooij ES. Tuning kinetics to control droplet shapes on chemically striped patterned surfaces. Langmuir. 2012;28:13137-13142.

26. Wang Y, Shangguan Q, Zhang D. Many-body dissipative particle dynamics simulation of the anisotropic effect of droplet wetting on stripe-patterned heterogeneous surfaces. Applied Surface Science. 2019;494:675-683.

27. Zou L, Wang H, Zhu X, Ding Y, Chen R, Liao Q. Droplet splitting on chemically striped surface. Colloids and Surfaces A: Physicochemical and Engineering Aspects. 2018;537:139-148. 
28. Cupelli C, Henrich B, Glatzel T, Zengerle R, Moseler M, Santer M. Dynamic capillary wetting studied with dissipative particle dynamics. New Journal of Physics. 2008;10:043009.

29. Liang Y-E, Weng Y-H, Hsieh IF, Tsao H-K, Sheng Y-J. Attractive encounter of a nanodrop toward a nanoprotrusion. The Journal of Physical Chemistry C. 2017;121:7923-7930.

30. Zhao J, Chen S, Liu Y. Spontaneous wetting transition of droplet coalescence on immersed micropillared surfaces. Applied Mathematical Modelling. 2018;63:390-404.

31. Zhang K, Li Z, Maxey M, Chen S, Karniadakis GE. Self-cleaning of hydrophobic rough surfaces by coalescence-induced wetting transition. Langmuir. 2019;35:2431-2442.

32. Wu C-J, Chu K-C, Sheng Y-J, Tsao H-K. Sliding dynamic behavior of a nanobubble on a surface. The Journal of Physical Chemistry C. 2017;121:17932-17940.

33. Plimpton S. Fast parallel algorithms for short-range molecular dynamics. Journal of Computational Physics. 1995;117: 1-19.

34. Warren PB. Vapor-liquid coexistence in many-body dissipative particle dynamics. Phys. Rev. E. 2003;68:066702.

35. Español P, Warren PB. Perspective: Dissipative particle dynamics. The Journal of Chemical Physics 2017, 146: 150901.

36. Hoogerbrugge PJ, Koelman JMVA. Simulating microscopic hydrodynamic phenomena with dissipative particle dynamics Europhysics Letters. 1992;19:155-160

37. Arienti M, Pan W, Li X, Karniadakis G. Many-body dissipative particle dynamics simulation of liquid/vapor and liquid/solid interactions. The Journal of chemical physics. $2011 ; 134: 204114$

38. Español P, Warren PB. Statistical mechanics of dissipative particle dynamics. Europhysics Letters. 1995;30:191-196. 
39. Son Y, Kim C. Spreading of inkjet droplet of non-Newtonian fluid on solid surface with controlled contact angle at low Weber and Reynolds numbers. Journal of Non-Newtonian Fluid Mechanics. 2009;162:78-87.

40. Vázquez UOM, Shinoda W, Moore PB, Chiu C-c, Nielsen SO. Calculating the surface tension between a flat solid and a liquid: a theoretical and computer simulation study of three topologically different methods. Journal of Mathematical Chemistry. 2008;45:161-174.

41. Backer JA, Lowe CP, Hoefsloot HC, Iedema PD. Poiseuille flow to measure the viscosity of particle model fluids. The Journal of chemical physics. 2005;122:154503.

42. Boromand A, Jamali S, Maia JM. Viscosity measurement techniques in dissipative particle dynamics. Computer Physics Communications. 2015;196:149-160.

43. Weng Y-H, Liang Y-E, Sheng Y-J, Tsao H-K. Controlling nanodrop passage through capillary nanovalves by adjusting lyophilic crevice structure. The Journal of Physical Chemistry C. 2018;122:2231-2237.

44. Ashoke Raman K, Jaiman R K, Lee Yong-See, Low Hong-Tong. Dynamics of simultaneously impinging drops on a dry surface: Role of impact velocity and air inertia. Journal of Colloid and Interface Science. 2017;486:265-276.

45. Ahmadlouydarab M, Hemeda AA, Ma Y. Six stages of microdroplet detachment from microscale fibers. Langmuir. 2018;34:198-204.

46. Lin S, Zhao B, Zou S, Guo J, Wei Z, Chen L. Impact of viscous droplets on different wettable surfaces: Impact phenomena, the maximum spreading factor, spreading time and post-impact oscillation. Journal of colloid and interface science. 2018;516:86-97.

47. Bennett T, Poulikakos D. Splat-quench solidification: estimating the maximum spreading of a droplet impacting a solid surface. Journal of Materials Science. 1993;28:963-970. 
48. Eggers J, Fontelos MA, Josserand C, Zaleski S. Drop dynamics after impact on a solid wall: Theory and simulations. Physics of Fluids. 2010;22:062101.

49. Clanet C, BÉGuin C, Richard D, QuÉRÉ D. Maximal deformation of an impacting drop. Journal of Fluid Mechanics. 2004;517:199-208.

50. Sarojini Kg K, Dhar P, Varughese S, Das SK. Coalescence dynamics of pedot:pss droplets impacting at offset on substrates for inkjet printing. Langmuir. 2016;32:5838-5851.

51. Chimene D, Peak CW, Gentry JL, et al. Nanoengineered ionic-covalent entanglement (nice) bioinks for 3d bioprinting. ACS Applied Materials \& Interfaces. 2018;10:9957-9968.

\section{Figure captions}

Figure 1. Schematic representation of a droplet on a chemically stripe-patterned surface: (a) Top view. (b) Front and side view. $W$ and $L$ represent the droplet width and length, respectively. $\theta_{\perp}$ and $\theta_{\|}$represent contact angles perpendicular and parallel to the direction of the stripes, respectively. (c) perspective view

Figure 2. Simulation validation (a) Radial density profile of the droplet (b) Velocity profile for periodic Poiseuille flow to calculate viscosity (c) Relationship between the solid-liquid interaction parameter $A_{s l}$ and the static contact angle $\theta_{\mathrm{c}}$. An error bar represents one standard deviation.

Figure 3. Simulation validation of time evolution of droplet dimensionless height $H^{*}$ and dimensionless diameter $D^{*}$ against experimental data reported in Ref. 39

Figure 4. Schematic illustration of droplet deposition on (a) the middle of hydrophobic stripe (Case 1) (b) the middle of hydrophilic stripe (Case 2) (c) the border between two different stripes (Case 3). The hydrophobic stripe width equals to hydrophilic stripe width, represented by $W_{\mathrm{s}}$

Figure 5. Evolution of droplet dimensionless width $W^{*}\left(W^{*}=W / D_{0}\right)$ and length $L^{*}\left(L^{*}=L / D_{0}\right)$ in (a) Case 1, (b) Case 2 and (c) Case 3. (d) Evolution of $L^{*}$ in Case 2 and Case 3 for a longer time. 
The inset in (a) shows the outline of "butterfly" shape as the $\theta_{\text {bic }}$ are $93^{\circ}$ (yellow line) and $115^{\circ}$ (blue line), respectively

Figure 6. Evolution of spreading droplet with zero Weber number in three deposition position cases

Figure 7. (a) $\theta_{\perp}$ and $\theta_{\|}$of the droplet at its equilibrium state dependence on hydrophobic stripe wettability $\left(A_{\text {bic }}\right)$ and $\beta$, i.e., the ratio of the stripe width and initial droplet diameter, when the intrinsic contact angle of hydrophilic stripes $\left(\theta_{\text {lic }}\right)$ is fixed as $45^{\circ}$. Pentagons represent the intrinsic contact angle of hydrophobic stripes $\left(\theta_{\text {bic }}\right)$ corresponding to $A_{\text {bic }}$ derived from Figure $2 \mathrm{c}$. (b) The channel like (left, $\beta=0.5, \theta_{\text {bic }}=170^{\circ}$ ) and bulge like (right, $\beta=0.8, \theta_{\text {bic }}=170^{\circ}$ ) shape. (c) $\theta_{\|}$ estimate example. The red curves are the polynomial fits. (see the text).

Figure 8. Aspect ratio of the droplet dependence on hydrophobic stripe wettability $A_{\text {bic }}\left(\theta_{\text {bic }}\right)$ and $\beta$

Figure 9. Split volume and maximum footprint area fractions of the droplet on two sides of a single stripe as the deposition position varies. $L$ is the distance between droplet deposition point and the center line of the stripe. $V_{\mathrm{f}, \mathrm{L}}=V_{\mathrm{L}} / V_{0}, V_{\mathrm{f}, \mathrm{R}}=V_{\mathrm{R}} / V_{0}, A_{\mathrm{f}, \mathrm{L}}=A_{\mathrm{L}} /\left(A_{\mathrm{L}}+A_{\mathrm{R}}\right), A_{\mathrm{f}, \mathrm{R}}=A_{\mathrm{R}} /\left(A_{\mathrm{L}}+A_{\mathrm{R}}\right)$, where $V_{\mathrm{L}}$ and $V_{\mathrm{R}}$ represent the left and right split droplet volume, $A_{\mathrm{L}}$ and $A_{\mathrm{R}}$ are the left and right footprint area when the droplet reaches its maximum spread on hydrophobic stripe

Figure 10. Evolution of impinging droplet with Weber number (We) equals 22.7 on single-stripe-patterned surface

Figure 11. (a) Determination of droplet split in a single-stripe system. The hollow circle signed the cases droplet split off, where $R_{\max }^{*}>\beta$ under the same We. The black solid squares represent $R_{\max }^{*}$ vs. We. (b) the logarithmic scale of $R_{\max }^{*}$ vs. We and they are the same as in the figure(a). (c) Time history of $R^{*}$ 
Figure 12. Temporal of impinging droplet with $W e=22.7$ on different $\beta$ surface. The time is in MDPD units

Figure 13. The scaled surface energy and kinetic energy of the droplet as a function of time for different $\beta$ surface. We fixed at 22.7, and the time $t_{1}, t_{2}, t_{3}, t_{4}, t_{5}$ referred back to Figure 12 .

Figure 14. (a) Droplet morphology as a function of $W e$ and $\beta$. The dot line represents the relationship of We and $R_{\max }^{*}$ derived from Figure 11a for single stripe system. (b) the scaled surface energy $E_{s}^{*}$ as a function of We

Figure 15. The relationship between $R_{\max }^{*}$ and $W e$ on homogenous surface with different intrinsic contact angle $\theta_{\text {bic }}$

Figure 16. Droplet morphology on multi-striped surface with (a) $\theta_{b i c}=170^{\circ} \theta_{\text {lic }}=45^{\circ}$ (b) $\theta_{b i c}=155^{\circ}$ $\theta_{l i c}=45^{\circ}$ (c) $\theta_{b i c}=170^{\circ} \theta_{l i c}=60^{\circ}$. The dotted lines in these three figures are the same, and its expression is $R_{\max }^{*}=0.41 W e^{0.20}$. The color coding is the same as in Fig. 14. 


\section{Figure 1}

(a)

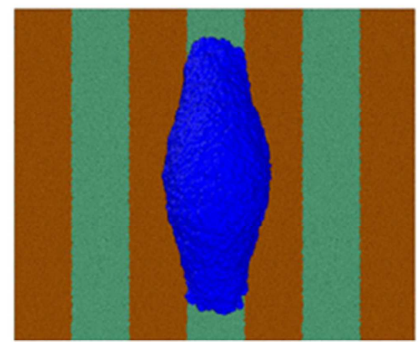

- hydrophobic

- hydrophilic (b)

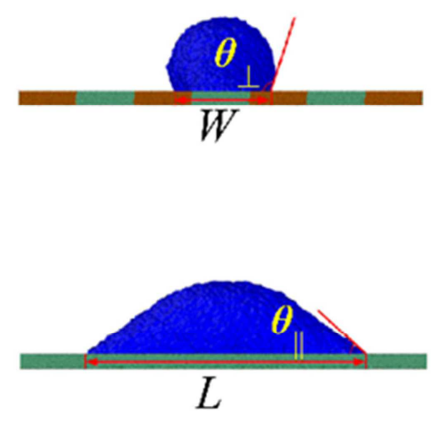

(c)

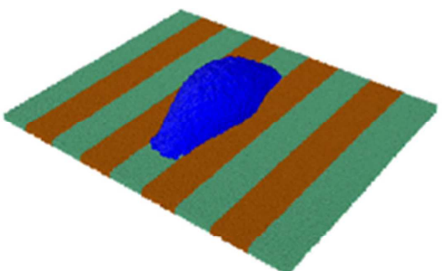

Figure 1. Schematic representation of a droplet on a chemically stripe-patterned surface: (a) Top view. (b) Front and side view. $W$ and $L$ represent the droplet width and length, respectively. $\theta_{\perp}$ and $\theta_{\|}$represent contact angles perpendicular and parallel to the direction of the stripes, respectively. (c) perspective view

\section{Figure 2}

(a)

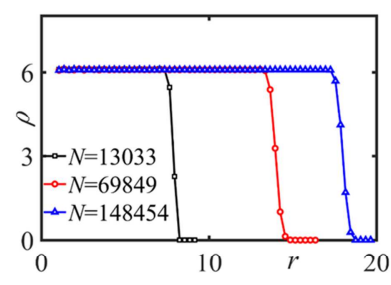

(b)

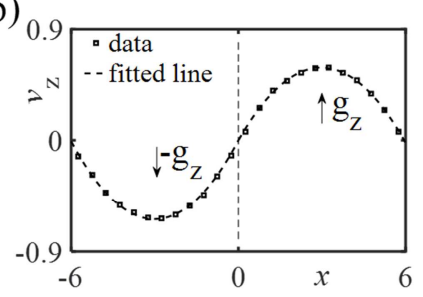

(c)

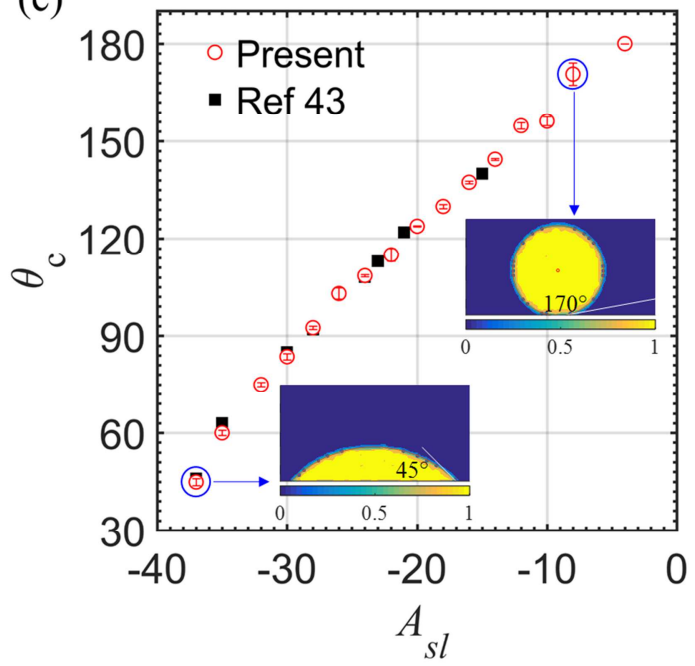

Figure 2. Simulation validation (a) Radial density profile of the droplet (b) Velocity profile for periodic Poiseuille flow to calculate viscosity (c) Relationship between the solid-liquid interaction parameter $A_{s l}$ and the static contact angle $\theta_{c}$. An error bar represents one standard deviation. 


\section{Figure 3}

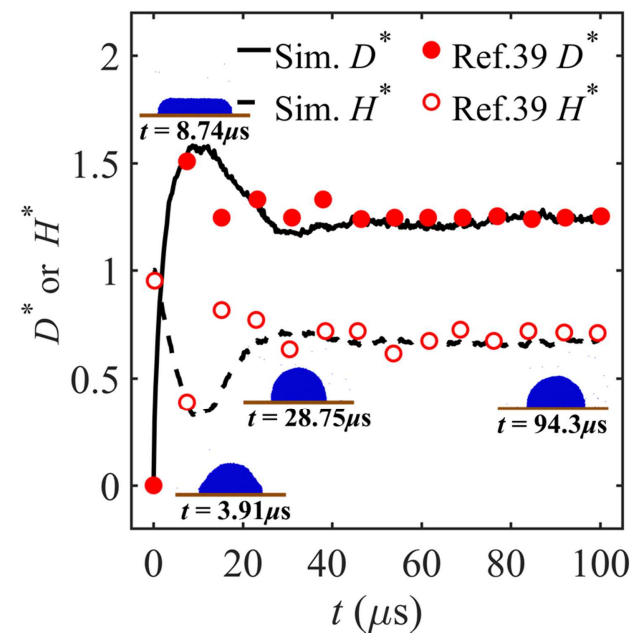

Figure 3. Simulation validation of time evolution of droplet dimensionless height $H^{*}$ and dimensionless diameter $D^{*}$ against experimental data reported in Ref. 39

\section{Figure 4}

(a)

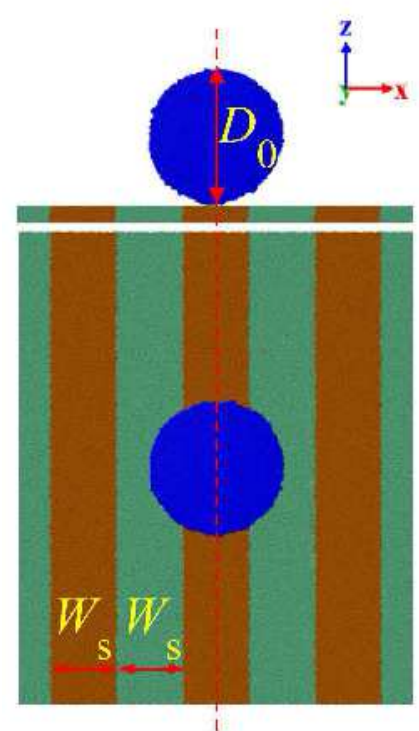

(b)

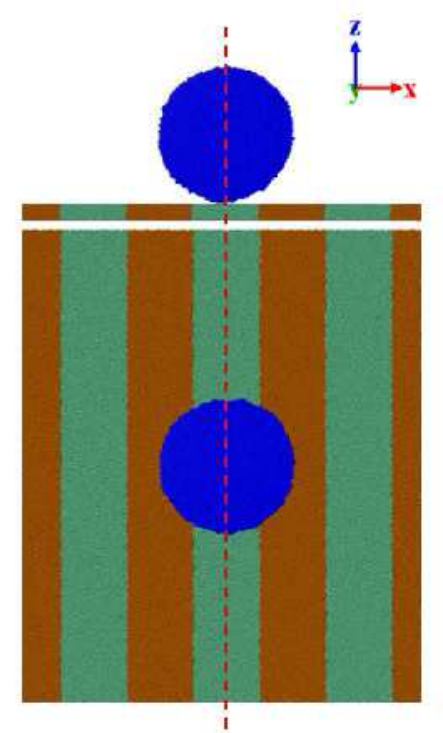

(c)

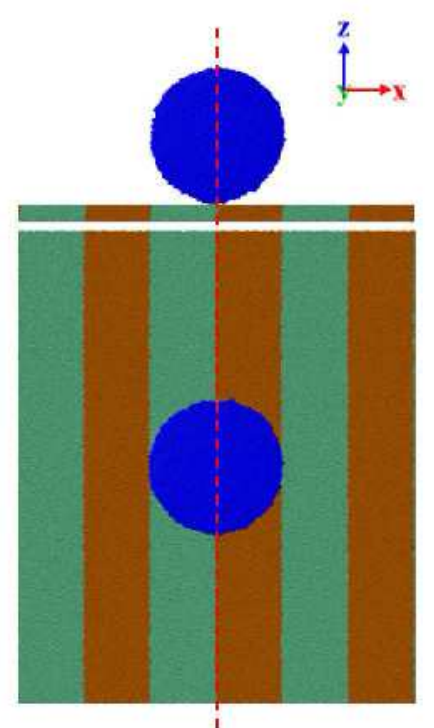

Figure 4. Schematic illustration of droplet deposition on (a) the middle of hydrophobic stripe (Case 1) (b) the middle of hydrophilic stripe (Case 2) (c) the border between two different stripes (Case 3). The hydrophobic stripe width equals to hydrophilic stripe width, represented by $W_{\mathrm{s}}$ 
Figure 5
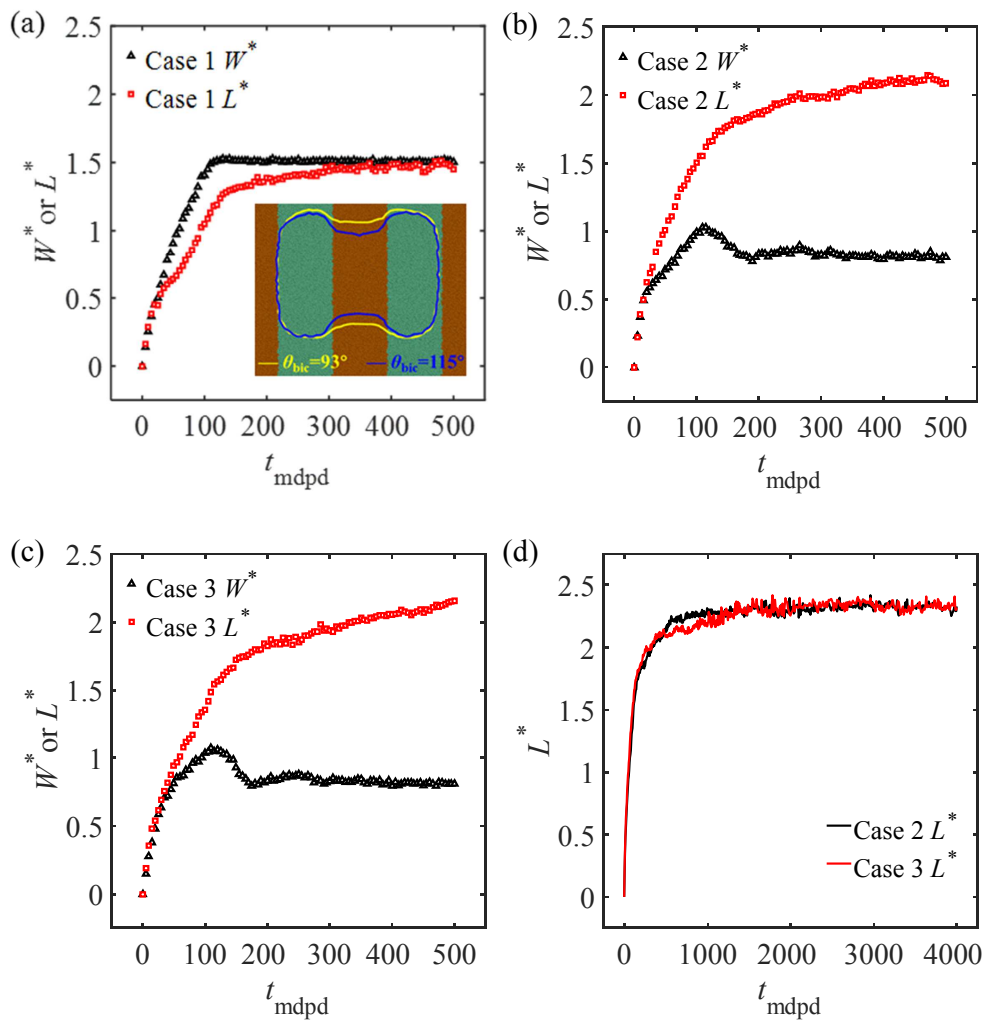

Figure 5. Evolution of droplet dimensionless width $W^{*}\left(W^{*}=W / D_{0}\right)$ and length $L^{*}\left(L^{*}=L / D_{0}\right)$ in (a) Case 1, (b) Case 2 and (c) Case 3. (d) Evolution of $L^{*}$ in Case 2 and Case 3 for a longer time. The inset in (a) shows the outline of "butterfly" shape as the $\theta_{\text {bic }}$ are $93^{\circ}$ (yellow line) and $115^{\circ}$ (blue line), respectively

\section{Figure 6}

(a) Case 1

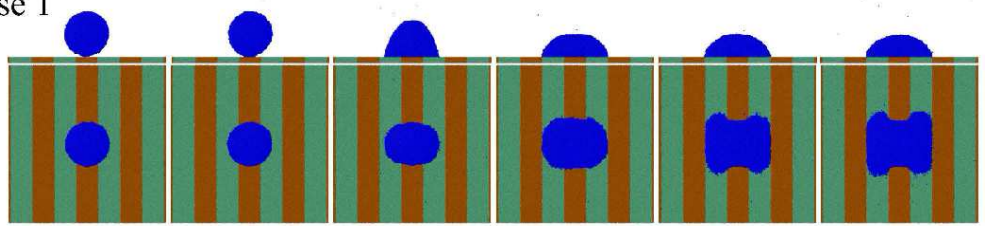

(b) Case 2

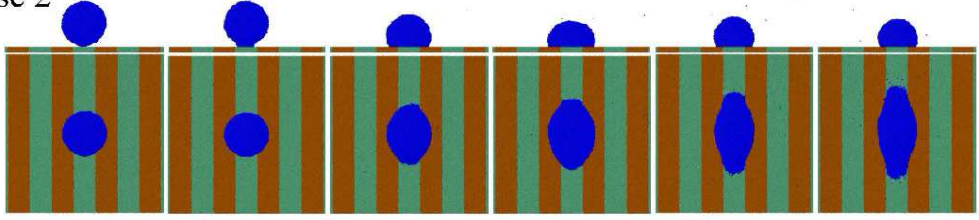

(c) Case 3

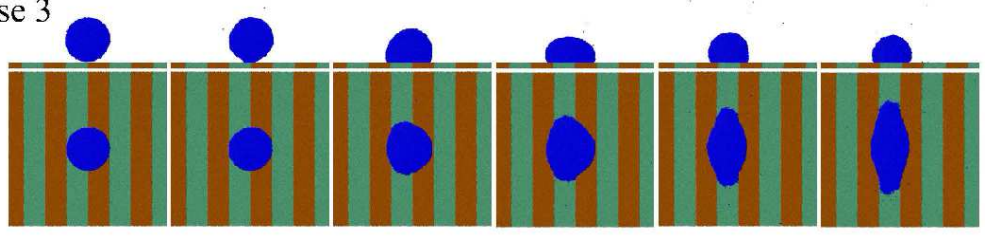

$$
t_{\text {mdpd }}=\begin{array}{llllll}
0 & 10 & 85 & 115 & 200 & 500
\end{array}
$$

Figure 6. Evolution of spreading droplet with zero Weber number in three deposition position cases 
Figure 7

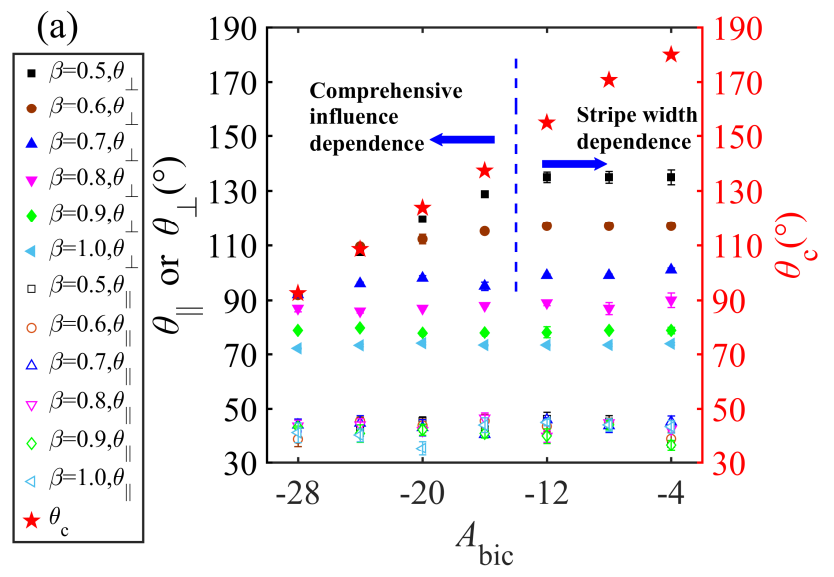

(b)

(c)
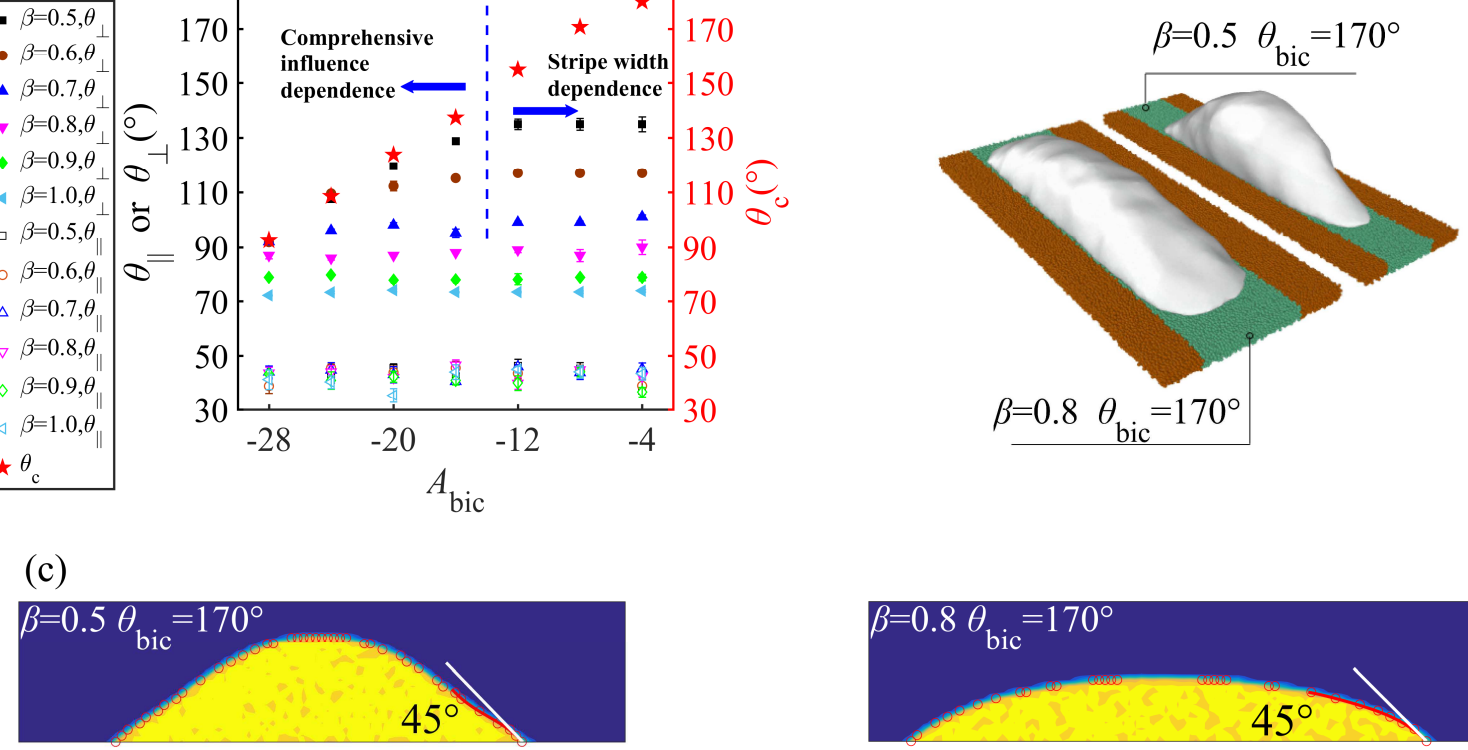

c)

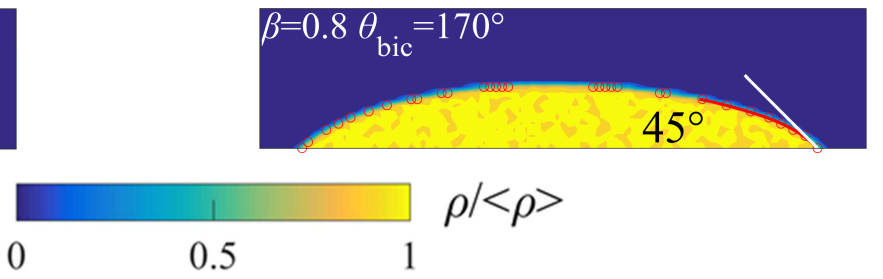

Figure 7. (a) $\theta_{\perp}$ and $\theta_{\|}$of the droplet at its equilibrium state dependence on hydrophobic stripe wettability $\left(A_{\text {bic }}\right)$ and $\beta$, i.e., the ratio of the stripe width and initial droplet diameter, when the intrinsic contact angle of hydrophilic stripes $\left(\theta_{\text {lic }}\right)$ is fixed as $45^{\circ}$. Pentagons represent the intrinsic contact angle of hydrophobic stripes $\left(\theta_{\text {bic }}\right)$ corresponding to $A_{\text {bic }}$ derived from Figure 2c. (b) The channel like (left, $\beta=0.5, \theta_{\text {bic }}=170^{\circ}$ ) and bulge like (right, $\beta=0.8, \theta_{\text {bic }}=170^{\circ}$ ) shape. (c) $\theta_{\|}$ estimate example. The red curves are the polynomial fits. (see the text).

\section{Figure 8}

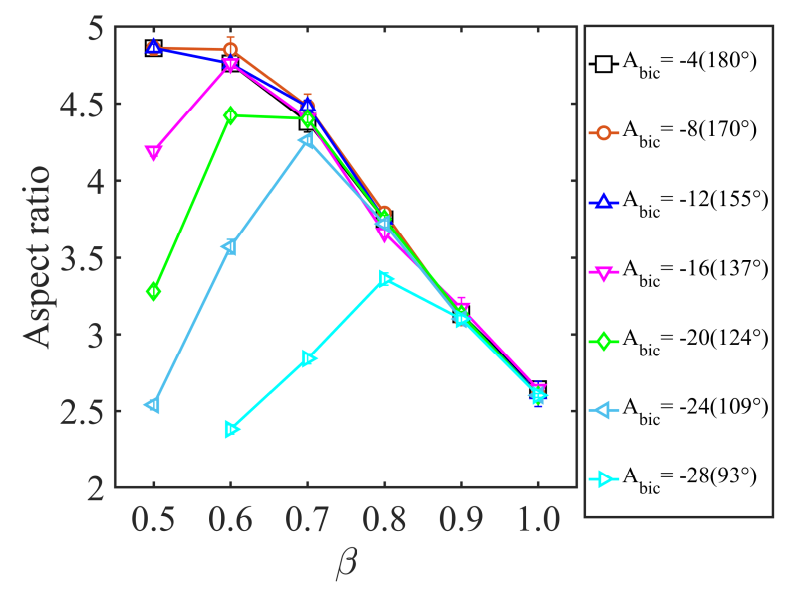

Figure 8. Aspect ratio of the droplet dependence on hydrophobic stripe wettability $A_{\text {bic }}\left(\theta_{\text {bic }}\right)$ and $\beta$ 


\section{Figure 9}

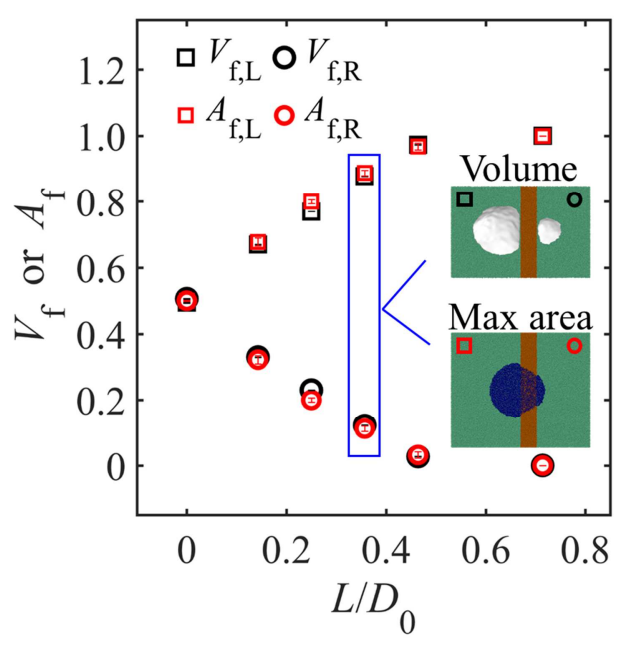

Figure 9. Split volume and maximum footprint area fractions of the droplet on two sides of a single stripe as the deposition position varies. $L$ is the distance between droplet deposition point and the center line of the stripe. $V_{\mathrm{f}, \mathrm{L}}=V_{\mathrm{L}} / V_{0}, V_{\mathrm{f}, \mathrm{R}}=V_{\mathrm{R}} / V_{0}, A_{\mathrm{f}, \mathrm{L}}=A_{\mathrm{L}} /\left(A_{\mathrm{L}}+A_{\mathrm{R}}\right), A_{\mathrm{f}, \mathrm{R}}=A_{\mathrm{R}} /\left(A_{\mathrm{L}}+A_{\mathrm{R}}\right)$, where $V_{\mathrm{L}}$ and $V_{\mathrm{R}}$ represent the left and right split droplet volume, $A_{\mathrm{L}}$ and $A_{\mathrm{R}}$ are the left and right footprint area when the droplet reaches its maximum spread on hydrophobic stripe

\section{Figure 10}

(a) $\beta=0.5$

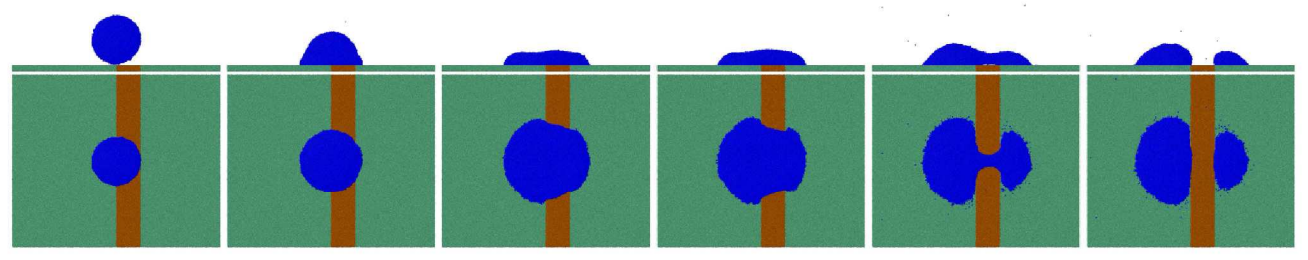

(b) $\beta=0.9$
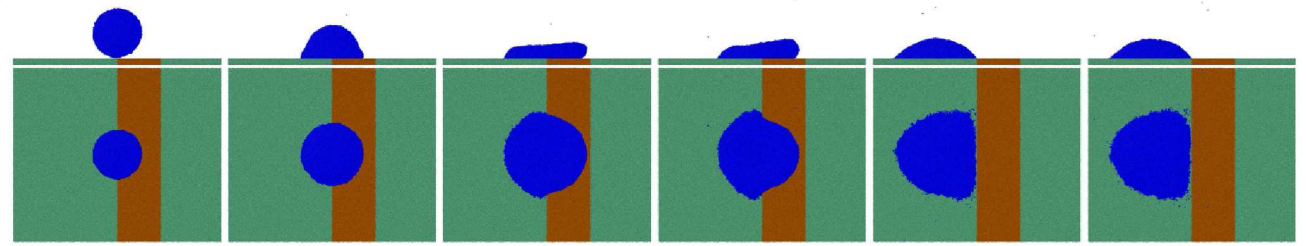

$$
t_{\text {mdpd }}=0
$$

10

30

40

200

230

Figure 10. Evolution of impinging droplet with Weber number (We) equals 22.7 on single-stripe-patterned surface 
Figure 11
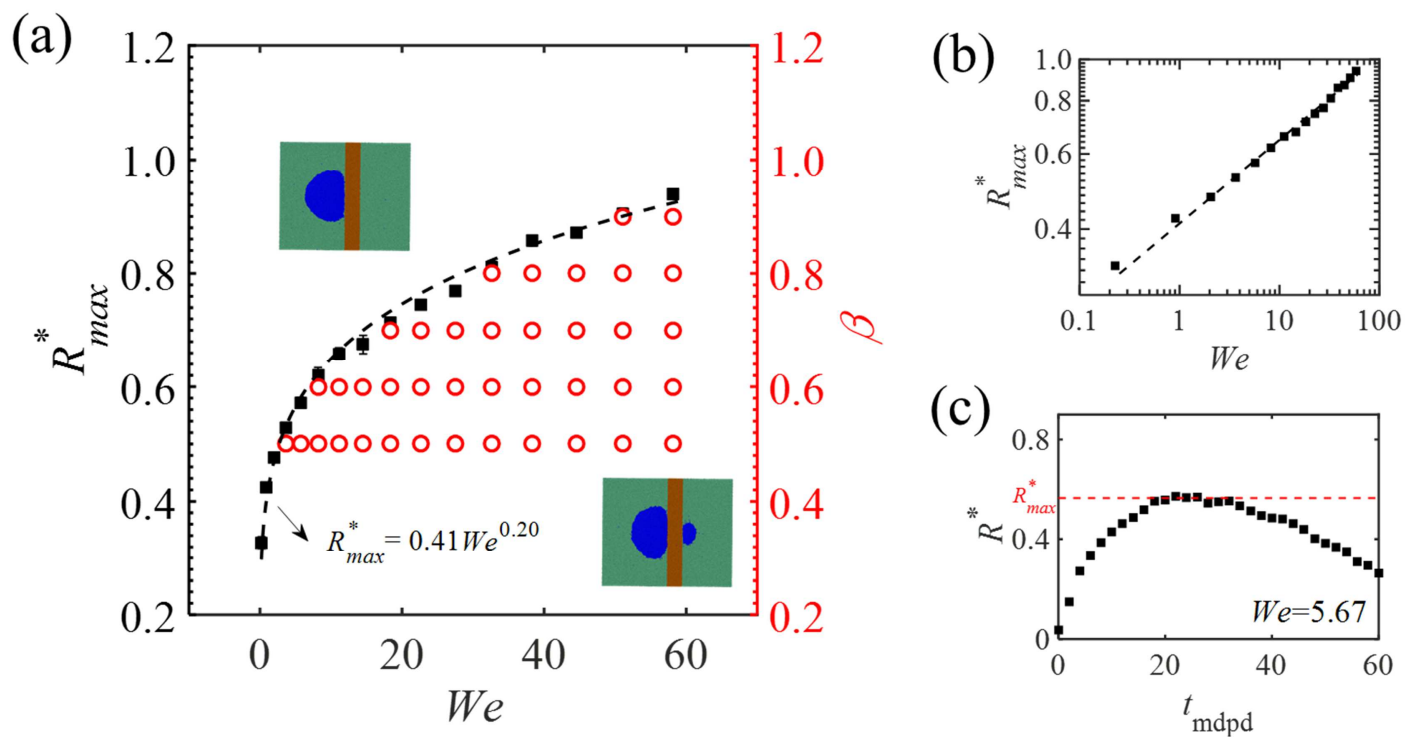

Figure 11. (a) Determination of droplet split in a single-stripe system. The hollow circle signed the cases droplet split off, where $R_{\max }^{*}>\beta$ under the same We. The black solid squares represent $R_{\max }^{*}$ vs. We. (b) the logarithmic scale of $R_{\max }^{*}$ vs. We and they are the same as in the figure(a). (c) Time history of $R^{*}$

\section{Figure 12}

(a) $\beta=0.5$

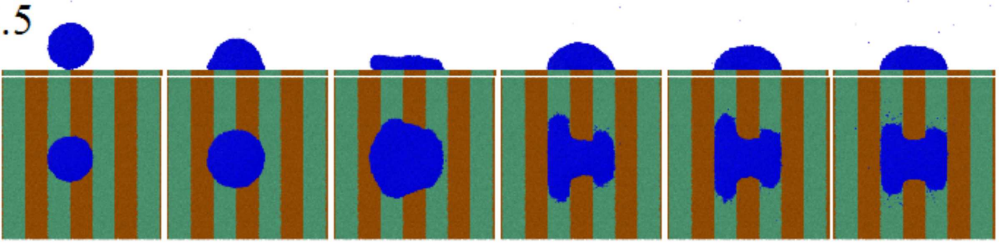

(b) $\beta=0.7$

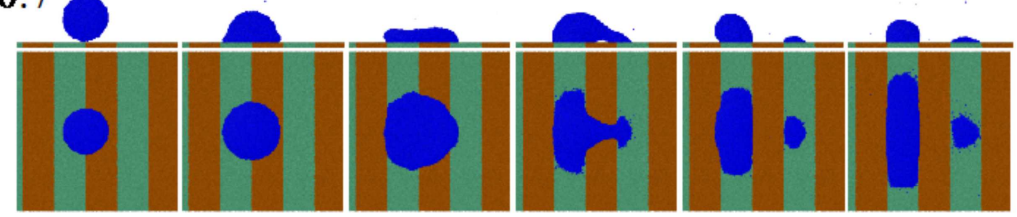

(c) $\boldsymbol{\beta}=\mathbf{0 . 9}$

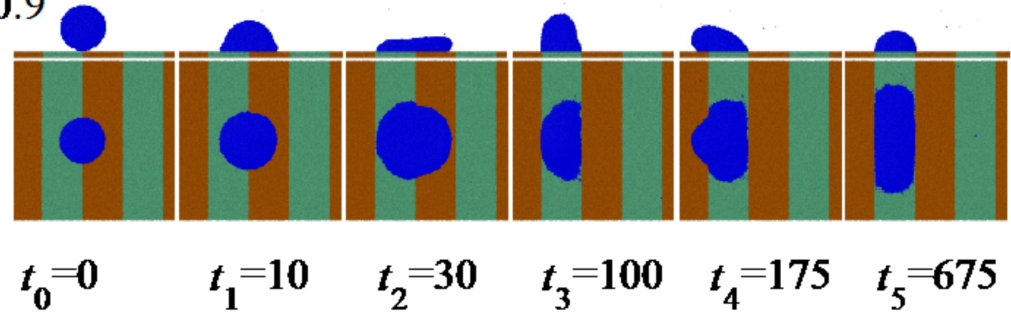

Figure 12. Temporal of impinging droplet with $W e=22.7$ on different $\beta$ surface. The time is in MDPD units 
Figure 13

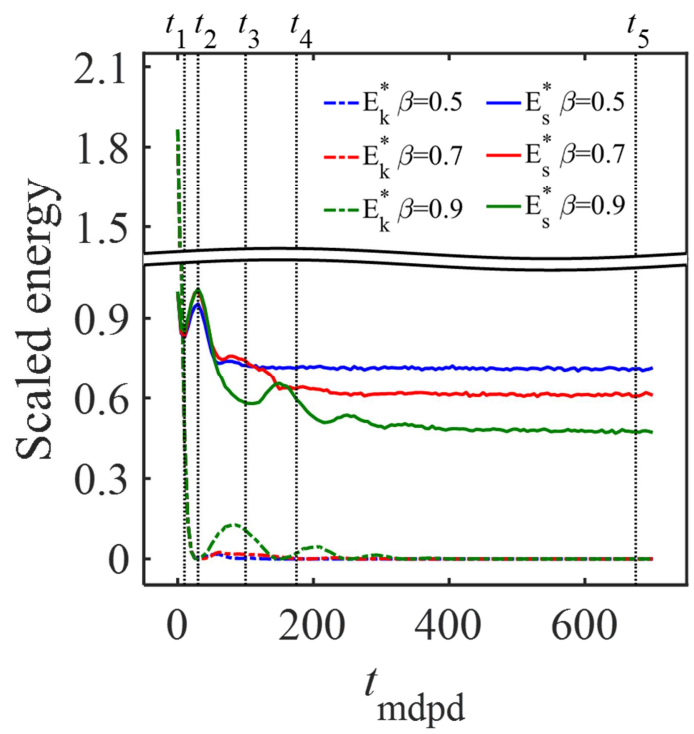

Figure 13. The scaled surface energy and kinetic energy of the droplet as a function of time for different $\beta$ surface. We fixed at 22.7, and the time $t_{1}, t_{2}, t_{3}, t_{4}, t_{5}$ referred back to Figure 12 .

\section{Figure 14}

(a)

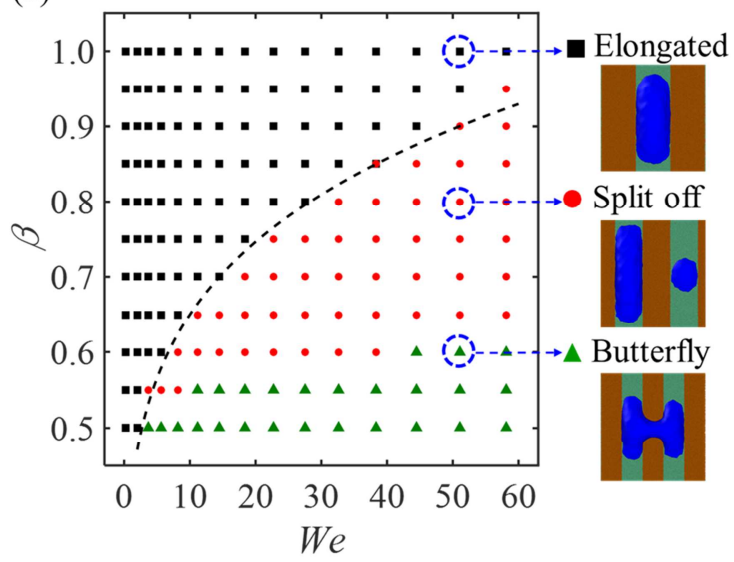

(b)

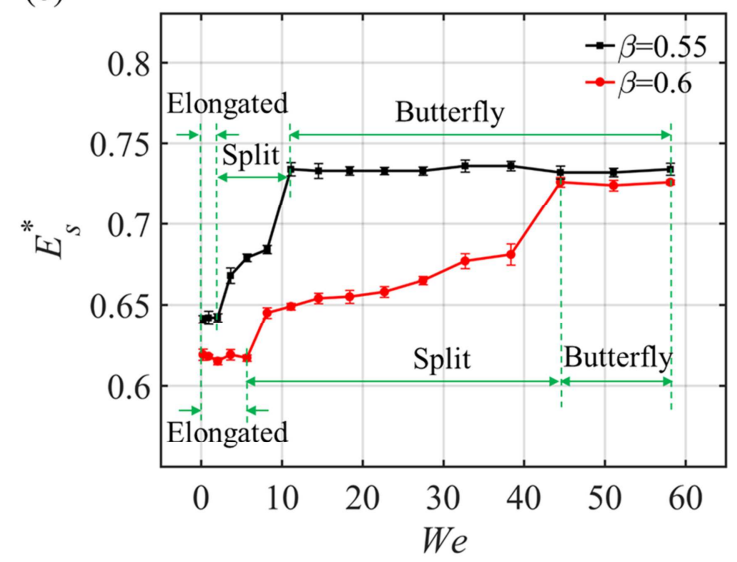

Figure 14. (a) Droplet morphology as a function of $W e$ and $\beta$. The dot line represents the relationship of We and $R_{\max }^{*}$ derived from Figure 11a for single stripe system. (b) the scaled surface energy $E_{s}^{*}$ as a function of We 


\section{Figure 15}

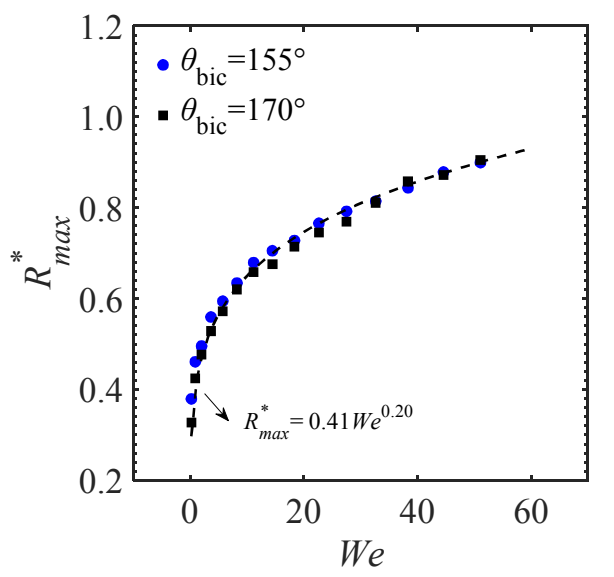

Figure 15. The relationship between $R_{\max }^{*}$ and $W e$ on homogenous surface with different intrinsic contact angle $\theta_{\text {bic }}$

\section{Figure 16}

(a)

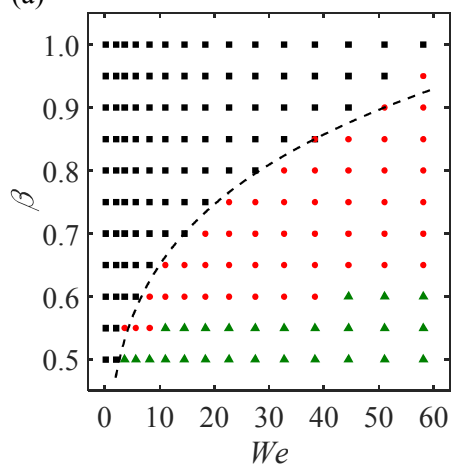

(b)

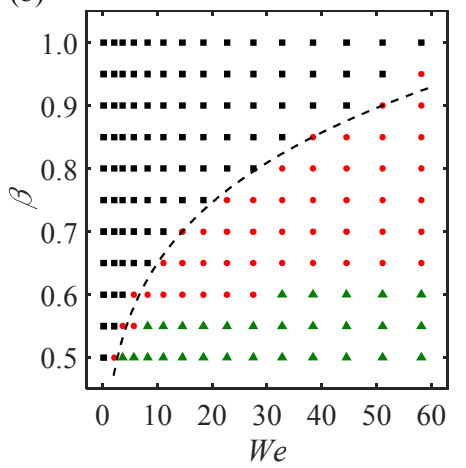

(c)

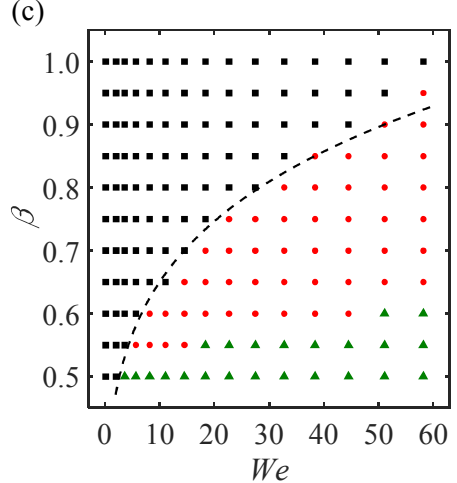

Figure 16 Droplet morphology on multi-striped surface with (a) $\theta_{b i c}=170^{\circ} \theta_{l i c}=45^{\circ}$ (b) $\theta_{b i c}=155^{\circ}$ $\theta_{l i c}=45^{\circ}$ (c) $\theta_{b i c}=170^{\circ} \theta_{\text {lic }}=60^{\circ}$. The dotted lines in these three figures are the same, and its expression is $R_{\max }^{*}=0.41 W e^{0.20}$. The color coding is the same as in Fig. 14 . 